\title{
QSRR Study of Linear Retention Indices for Volatile Compounds using Statistical Methods
}

\author{
ASSIA BELHASSAN ${ }^{1,2}$, SAMIR CHTITA $^{1}$, \\ TAHAR LAKHLIFI $^{1}$ and MOHAMMED BOUACHRINE ${ }^{2 *}$ \\ ${ }^{1}$ MCNS Laboratory, Faculty of Science, Moulay Ismail University, Meknes, Morocco \\ ${ }^{2}$ Materials, Environment and Modeling Laboratory, (ESTM) High School of Technology, \\ Moulay Ismail University, Meknes, Morocco \\ m.bouachrine@est-umi.ac.ma
}

Received 7 January 2018 / Accepted 30 January 2018

\begin{abstract}
ACD/ChemSketch, MarvinSketch and ChemOffice programs were used to calculate several molecular descriptors of 138 volatile compounds (32 hydrocarbons, 29 ketones, 28 aldehydes, 23 alcohols, 7 carboxylic acids, 6 halogenated compounds, 4 furans, 2 pyrazines, 1 ester, 1 sulphur compounds, 1 pyridine, 1 amine and three other compounds). The best descriptors were selected to establish the quantitative structure retention relationship (QSRR) of linear retention indices of volatile compounds using multiple linear regressions (MLR), multiple non-linear regressions (MNLR) and artificial neural network (ANN) methods. We propose quantitative models according to these analyses. The models were used to predict the linear retention indices of the test set compounds and agreement between the experimental and predicted values was verified. The descriptors showed by QSRR study were used for study and designing of new compounds. The statistical results indicate that the predicted values are in good agreement with the experimental results. To validate the predictive power of the resulting models, external validation multiple correlation coefficient was calculated and has both in addition to a performance prediction power, a favorable estimation of stability.
\end{abstract}

Keywords: Volatile compounds, Linear retention indices, Quantitative structure retention relationship, Multiple linear regression, Artificial neural network

\section{Introduction}

Retention is a phenomenon primarily dependent on the interactions between the solute and the stationary phase of molecules, which included directional force, induction force, dispersion force and hydrogen bond ${ }^{1}$. These forces can be related to the structure of molecules; therefore, it was possible to predict the solute retention from molecular descriptors. Quantitative structure retention relationships (QSRR) studies have received much attention in chemometrics, biological chemistry, medicinal chemistry and many other fields. QSRR models are mathematical equations relating chemical structure to their property. 
A number of reports that deals with QSRR retention calculation of several compounds have been published in the literature ${ }^{2,3}$. QSRR has been demonstrated to be a powerful tool for the investigation of the chromatographic parameters. In QSRR approaches, the structural features of solutes encoded by chemical descriptors and then the relationship between these descriptors and solute retention were studied ${ }^{4}$.

The QSRR models can be applied to multiple linear regressions (MLR). Because of the complexity of relationships between the property of molecules and structures, nonlinear models are also used to model the structure-property relationships. Multiple non-linear regression (MNLR) and artificial neural network (ANN) methods are nonparametric nonlinear modeling technique that have attracted increasing interest.

The purpose of this work is to study the linear retention indices (LRI) of volatile compounds using QSRR chemical modeling methods. The main steps in this method includes: data collection, molecular descriptors generation, descriptor selection, model development and finally model performance evaluation ${ }^{5}$.

\section{Experimental}

In this study, we selected 138 volatile compounds with linear retention indices (LRI) reported in the literature ${ }^{6}$ to provide a diversified set of chemical families (hydrocarbons, alcohols, aldehydes, ketones, esters etc. The list of molecules and the $\log (\mathrm{LRI})$ values are listed in Table 1.

A total of 97 molecules were placed in the training set to build the QSRR models, whereas the remaining, 41 molecules constitute the test set. The division was carried out by random selection using the SPSS 19.0 statistical package ${ }^{7}$.

\section{Molecular descriptors}

A wide variety of molecular descriptors were calculated using ACD/ChemSketch, MarvinSketch and ChemOffice software $e^{8-10}$ to predict the correlation between these descriptors and the linear retention indices of the molecules studied (Table 2). Tables S1 show values of these descriptors for each molecule studied.

\section{Statistical analysis}

Multiple linear regression (MLR) is used to study the relationship between a dependent variable and several independent variables; it minimizes the differences between actual and predicted values and has been used to select the descriptors to be used as inputs in multiple non-linear regression (MNLR) and artificial neural network ANN. Linear and non-linear approaches were used to predict the effects on the linear retention indices, the equations were justified by the correlation coefficient $(r)$, the mean square error $(M S E)$, the fisher value $(F)$ and the significance level $(p)^{11}$. MLR, MNLR and ANN are generated using the SPSS 19.0 statistical package ${ }^{7}$.

Cross-validations, the most commonly used techniques for internal validation, are statistical techniques in which different proportions of chemicals are iteratively held-out from the training set used for model development (an optimal parameters $K$ selection step) and "predicted" as new by the developed model in order to verify internal "predictivity". In this work the Leave-One-Out is used, this procedure successively removes one molecule from the training set containing 97 molecules. A QSRR model is constructed on a "96" set of compounds and the molecule removed is predicted by the model. This procedure is repeated "97" times in order to predict the property of all molecules ${ }^{12}$. 
Table 1. List of aroma compounds

\begin{tabular}{|c|c|c|c|c|c|}
\hline $\mathrm{N}^{\circ}$ & Molecule name & $\log (\mathrm{LRI})$ & $\mathrm{N}^{\circ}$ & Molecule name & $\log (\mathrm{LRI})$ \\
\hline 1 & Octane & 2.904 & $42^{\mathrm{a}}$ & 2,3-Butanedione & 2.990 \\
\hline 2 & Nonane & 2.954 & 43 & 2,5-Octadione & 3.124 \\
\hline 3 & Pentadecane & 3.175 & 44 & (Z,E)-3,5-Octadien-2-one & 3.187 \\
\hline 4 & Heptadecane & 3.066 & 45 & (E,E)-3,5-Octadien-2-one & 3.202 \\
\hline 5 & 2-Methylheptane & 2.875 & $46^{\mathrm{a}}$ & 3,5-Dimethyl-2,7-octadione & 3.277 \\
\hline $6^{\mathrm{a}}$ & 4-Methylheptane & 2.879 & 47 & 6-Methyl-3,5-heptadien-2-one & 3.208 \\
\hline 7 & 4-Methyloctane & 2.931 & 48 & 6-Methyl-5-hepten-2-one & 3.131 \\
\hline 8 & 2-Methylnonane & 2.979 & $49^{\mathrm{a}}$ & 1-Hydroxy-2-propanone & 3.120 \\
\hline 9 & 4-Methyldecane & 3.023 & 50 & 3-Hydroxy-2-butanone & 3.115 \\
\hline 10 & 5-Methyldecane & 3.021 & 51 & 2,2,6-Trimethylcyclohexanone & 3.128 \\
\hline 11 & 2-Methylundecane & 3.063 & $52^{\mathrm{a}}$ & $\begin{array}{c}\text { 3,5,5-Trimethyl-2-cyclohexen- } \\
\text { 1-one }\end{array}$ & 3.156 \\
\hline 12 & 3-Methylundecane & 3.092 & 53 & 2-Propyl-2-cyclohexenone & 3.170 \\
\hline 13 & 4-Ethyldecane & 3.014 & 54 & $\gamma$-Valerolactone & 3.216 \\
\hline 14 & 2,4-Dimethylhexane & 2.975 & 55 & $\gamma$-Butyrolactone & 3.222 \\
\hline $15^{\mathrm{a}}$ & 2,4-Dimethylheptane & 2.906 & 56 & 1-Phenylethanone & 3.226 \\
\hline $16^{\mathrm{a}}$ & 2,7-Dimethyloctane & 2.960 & 57 & $\gamma$-Hexalactone & 3.240 \\
\hline 17 & 2,5-Dimethylnonane & 3.000 & $58^{\mathrm{a}}$ & 5-Ethyl-2(5H)-furanone & 3.254 \\
\hline 18 & 3,7-Dimethyldecane & 3.034 & 59 & 4-Ketoisophorone & 3.237 \\
\hline $19^{\mathrm{a}}$ & 4,4-Dimethylundecane & 2.998 & 60 & $\alpha$-Ionone & 3.276 \\
\hline 20 & 2,4-Dimethylundecane & 3.076 & 61 & $\beta$-Ionone & 3.296 \\
\hline 21 & 2,6-Dimethylundecane & 3.077 & 62 & Ethanal & 2.848 \\
\hline $22^{\mathrm{a}}$ & 4,8-Dimethylundecane & 3.082 & 63 & Propanal & 2.902 \\
\hline 23 & 4,6-Dimethyldodecane & 3.088 & $64^{\mathrm{a}}$ & Butanal & 2.946 \\
\hline 24 & 2,4,6-Trimethyloctane & 3.085 & 65 & Pentanal & 2.994 \\
\hline 25 & (E)-2-Octene & 2.940 & 66 & Hexanal & 3.040 \\
\hline $26^{\mathrm{a}}$ & 8-Heptadecene & 3.237 & 67 & Heptanal & 3.078 \\
\hline 27 & 2,4-Dimethyl-1-heptene & 2.946 & 68 & Octanal & 3.115 \\
\hline 28 & 1,4-Octadiene & 3.268 & 69 & Nonanal & 3.149 \\
\hline 29 & 1,3-Cyclooctadiene & 3.229 & 70 & Decanal & 3.180 \\
\hline $30^{\mathrm{a}}$ & $\begin{array}{l}\text { 3-Ethyl-2-methyl-1,3- } \\
\text { hexadiene }\end{array}$ & 3.160 & $71^{\mathrm{a}}$ & (E)-2-Butenal & 3.023 \\
\hline 31 & 1,3-Dimethylbenzene & 3.065 & 72 & (E)-2-Pentenal & 3.060 \\
\hline 32 & 1,2,3-Trimethylbenzene & 3.120 & 73 & (E)-2-Hexenal & 3.092 \\
\hline 33 & 2-Propanone & 2.913 & 74 & (E)-2-Heptenal & 3.128 \\
\hline 34 & 2-Butanone & 2.960 & 75 & (Z)-4-Heptenal & 3.099 \\
\hline $35^{\mathrm{a}}$ & 2-Pentanone & 2.994 & $76^{\mathrm{a}}$ & (E)-2-Octenal & 3.161 \\
\hline $36^{\mathrm{a}}$ & 3-Hexanone & 3.027 & $77^{\mathrm{a}}$ & (Z,E)-2,4-Heptadienal & 3.173 \\
\hline $37^{\mathrm{a}}$ & 2-Heptanone & 3.077 & $78^{\mathrm{a}}$ & (E,E)-2,4-Heptadienal & 3.181 \\
\hline 38 & 2-Octanone & 3.113 & 79 & (E,E)-2,6-Nonadienal & 3.207 \\
\hline 39 & 3-Penten-2-one & 3.058 & 80 & 2-Methylbutanal & 2.964 \\
\hline $40^{\mathrm{a}}$ & 1-Octen-3-one & 3.119 & 81 & 3-Methylbutanal & 2.966 \\
\hline 41 & 3-Octen-2-one & 3.131 & $82^{\mathrm{a}}$ & 2-Methyl-2-propenal & 2.947 \\
\hline
\end{tabular}

Contd... 


\begin{tabular}{|c|c|c|c|c|c|}
\hline $\mathrm{N}^{\circ}$ & Molecule name & $\log (\mathrm{LRI})$ & $\mathrm{N}^{\circ}$ & Molecule name & $\log (\mathrm{LRI})$ \\
\hline $83^{\mathrm{a}}$ & (E)-2-Methyl-2-butenal & 3.046 & $111^{\mathrm{a}}$ & 1,2-Dimethylcyclohexanol & 3.214 \\
\hline 84 & 2-Methyl-2-pentenal & 3.072 & 112 & Benzenemethanol & 3.279 \\
\hline 85 & Benzaldehyde & 3.191 & $113^{\mathrm{a}}$ & Ethanoic acid & 3.164 \\
\hline $86^{\mathrm{a}}$ & 4-Ethylbenzaldehyde & 3.241 & $114^{\mathrm{a}}$ & Butanoic acid & 3.215 \\
\hline $87^{\mathrm{a}}$ & 4-Propylbenzaldehyde & 3.272 & 115 & Hexanoic acid & 3.274 \\
\hline 88 & $\beta$-Cyclocitral & 3.220 & 116 & Heptanoic acid & 3.298 \\
\hline 89 & Safranal & 3.226 & 117 & 2-Propenoic acid & 3.216 \\
\hline 90 & 1-Pentanol & 3.100 & 118 & 2-Methylpropanoic acid & 3.198 \\
\hline $91^{\mathrm{a}}$ & 3-Pentanol & 2.957 & 119 & 3-Methylbutanoic acid & 3.230 \\
\hline 92 & 1-Hexanol & 3.133 & $120^{\mathrm{a}}$ & Lactic acid ethyl ester & 3.131 \\
\hline 93 & 1-Octanol & 3.194 & 121 & Iodo-methane & 2.910 \\
\hline 94 & 1-Penten-3-ol & 3.068 & $122^{\mathrm{a}}$ & Iodo-ethane & 2.947 \\
\hline 95 & (E)-2-Penten-1-ol & 3.120 & 123 & 2-Iodo-propane & 2.960 \\
\hline $96^{\mathrm{a}}$ & (Z)-2-Penten-1-ol & 3.123 & 124 & 1-Iodo-pentane & 3.072 \\
\hline 97 & 1-Octen-3-ol & 3.162 & 125 & 1-Iodo-octane & 3.178 \\
\hline 98 & 2-Octen-1-ol & 3.210 & 126 & 5-Chloro-1-pentene & 3.012 \\
\hline 99 & 2,3-Butanediol & 3.187 & $127^{\mathrm{a}}$ & Dimethyl sulphide & 2.876 \\
\hline $100^{\mathrm{a}}$ & (Z)-1,5-Octadien-3-ol & 3.173 & 128 & 2-Methylfuran & 2.942 \\
\hline 101 & 1,7-Octadien-3-ol & 3.179 & 129 & 2-Ethylfuran & 2.981 \\
\hline 102 & 3,5-Octadien-2-ol & 3.154 & $130^{\mathrm{a}}$ & 2-Pentylfuran & 3.095 \\
\hline 103 & 2-Methyl-3-pentanol & 3.134 & 131 & cis-2-(2-Pentenyl)furan & 3.118 \\
\hline $104^{\mathrm{a}}$ & (E)-2-Ethyl-1-hexanol & 3.251 & 132 & 2.6-Dimethylpyrazine & 3.129 \\
\hline 105 & (Z)-2-Ethyl-1-hexanol & 3.175 & $133^{\mathrm{a}}$ & $\begin{array}{c}2,3,5,6- \\
\text { Tetramethylpyrazine }\end{array}$ & 3.176 \\
\hline 106 & $\begin{array}{l}\text { 2-(2-Methoxypropoxy)-1- } \\
\text { propanol }\end{array}$ & 3.173 & $134^{\mathrm{a}}$ & 2-Ethylpyridine & 3.116 \\
\hline 107 & $\begin{array}{l}\text { 1-(2-Methoxypropoxy)-2- } \\
\text { propanol }\end{array}$ & 3.187 & 135 & $\begin{array}{l}\text { N,N-Dimethyl- } \\
\text { methanamine }\end{array}$ & 2.796 \\
\hline 108 & $\begin{array}{l}\text { 1-(2-Methoxy-1-methyl- } \\
\text { ethoxy)-2-propanol }\end{array}$ & 3.181 & $136^{\mathrm{a}}$ & $\begin{array}{c}\text { 1-Acetyl-2,2- } \\
\text { dimethylcyclobutane } \\
\text { 1-Methoxy-4-(1'- }\end{array}$ & 3.006 \\
\hline $109^{\mathrm{a}}$ & 1-Ethoxy-2-propanol & 3.069 & 137 & $\begin{array}{l}\text { methylethyl)cyclohexa- } \\
\text { 1,4-diene }\end{array}$ & 3.300 \\
\hline $110^{\mathrm{a}}$ & Cyclooctanol & 3.209 & 138 & $\begin{array}{c}\text { 2,7-Epoxy-megastigma- } \\
\text { 4,8-diene }\end{array}$ & 3.178 \\
\hline
\end{tabular}


$Y$-randomization, randomly scrambling the responses, is another internal validation approach that must be used in parallel with cross-validations, and must always be applied to test the significance of the derived QSRR model, highlighting the presence of apparent models, obtained only by chance correlation ${ }^{12}$. We performed in this work 100-yrandomization tests for the MLR and MNLR models. In this test, random QSPR models are generated by randomly shuffling the dependent variable while keeping the independent variables as it is. The new QSRR models are expected to have significantly low $\mathrm{r}^{2}$ and $\mathrm{r}_{\mathrm{CV}}^{2}$ values for several trials, which confirm that the developed QSRR models are robust.

Table 2. Calculated chemical descriptors

\begin{tabular}{clc}
\hline \multicolumn{1}{c}{ Descriptors } & Abbreviation \\
\hline Software & \multicolumn{1}{c}{$H^{\circ}$} & $G$ \\
& Heat of formation, $\mathrm{kJ} \mathrm{mol}^{-1}$ & $\mathrm{IGTC}$ \\
& Gibbs free energy, $\mathrm{kJ} \mathrm{mol}^{-1}$ & $T$ \\
& Ideal gas thermal capacity, $\mathrm{J} \mathrm{mol}^{-1} \mathrm{~K}^{-1}$ & $\mathrm{CT}$ \\
& Melting point(Kelvin) & $\mathrm{TB}$ \\
& Critical temperature, $\mathrm{K}$ & $\mathrm{CP}$ \\
& Boiling point(Kelvin) & $\mathrm{KH}$ \\
ChemOffice & Critical pressure(Bar) & $\mathrm{TVC}$ \\
& Henry's law constant & $\mathrm{PC}$ \\
& Total valence connectivity & $\mathrm{NRB}$ \\
& Partition coefficient & $I$ \\
& Number of rotatable bonds & $\mathrm{SVD}$ \\
& Shape coefficient & $\mathrm{TC}$ \\
& Sum of valence degrees & $\mathrm{H} \%$ \\
& Total connectivity & $\mathrm{O} \%$ \\
& Percent ratios of hydrogen & $\mathrm{C} \%$ \\
& Percent ratios of oxygen & $\gamma$ \\
& Percent ratios of carbon & $n$ \\
& Surface tension & $d$ \\
& Index of refraction & Log $P$ \\
& Density & $W$ \\
& Log $P$ & $\mathrm{NHA}$ \\
& Winner index & NHD \\
& Number of H-Bond acceptors & $J$ \\
& Number of H-Bond donors & PSA \\
& Balaban index &
\end{tabular}

\section{Results and Discussion}

Data set for analysis

A QSRR study was carried out for a series of 138 volatile compounds, as indicated above, to determine a quantitative relationship between the structure and the linear retention indices for molecules studied. The values of the 27 descriptors are shown in Table S1.

\section{Multiple linear regressions (MLR)}

In order to propose a mathematical model and to evaluate quantitatively the substituent's physicochemical effects on the $\log (\mathrm{LRI})$ of the totality of the set of these 138 molecules, we submitted the data matrix constituted obviously from the 27 variables corresponding to the 97 molecules (training set), to a stepwise multiple regression analysis. 
Table S1. Chemical descriptors computed by ChemOffice, ChemSketch and MarvinSketch software

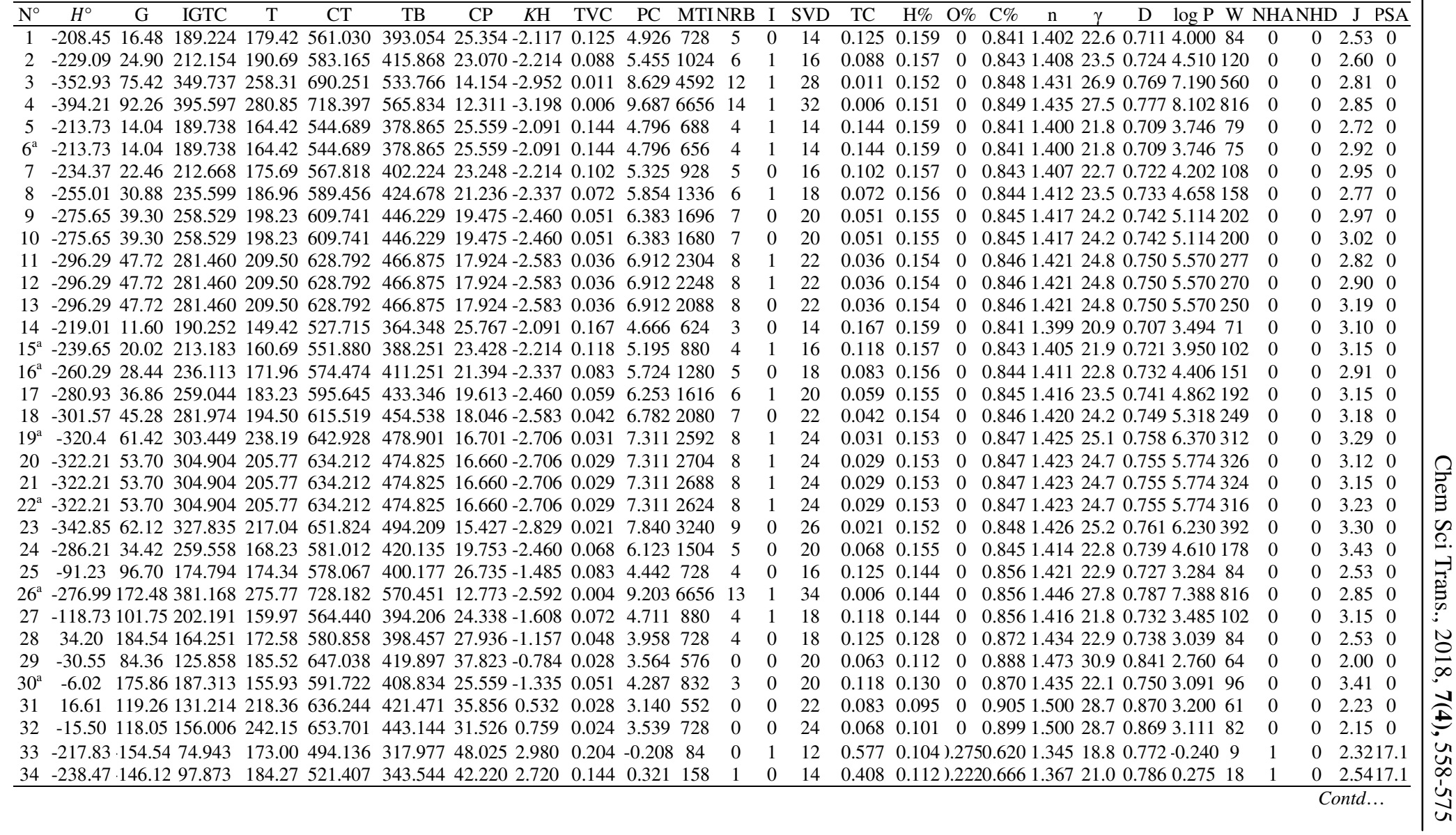




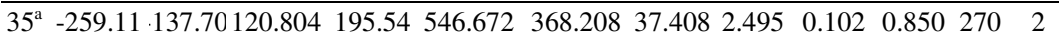
$\begin{array}{lllllllllll}36^{\mathrm{a}} & -279.75 & 129.28143 .734 & 206.81 & 570.140 & 391.968 & 33.374 & 2.324 & 0.072 & 1.379 & 416\end{array}$ $\begin{array}{llllllllllll}37^{\mathrm{a}} & -300.39 & 120.86166 .665 & 218.08 & 591.988 & 414.823 & 29.959 & 2.210 & 0.051 & 1.908 & 640 & 4\end{array}$ $\begin{array}{llllllllllll}38 & -321.03 & 112.44 & 189.595 & 229.35 & 612.372 & 436.775 & 27.042 & 2.100 & 0.036 & 2.437 & 914\end{array}$ $\begin{array}{llllllllllll}39 & -141.89 & -57.48 & 106.374 & 190.46 & 565.427 & 375.623 & 39.906 & 3.062 & 0.068 & 0.516 & 270\end{array}$ $\begin{array}{lllllllllllll}40^{\mathrm{a}} & -195.60 & -24.60 & 179.052 & 227.59 & 615.067 & 435.166 & 28.263 & 2.715 & 0.021 & 2.103 & 890 & 5\end{array}$ $\begin{array}{lllllllllllll}41 & -203.81 & -32.22 & 175.165 & 224.27 & 628.530 & 443.355 & 28.566 & 2.702 & 0.024 & 2.103 & 914 & 4\end{array}$

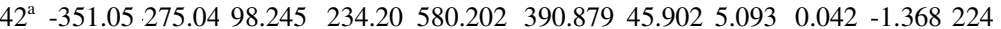
$\begin{array}{lllllllllll}43 & -433.61 & 241.36189 .966 & 279.28 & 657.834 & 477.047 & 28.905 & 5.496 & 0.010 & 0.905 & 1084\end{array}$ $\begin{array}{llllllllllll}44 & -86.59 & 48.00 & 160.736 & 219.19 & 645.194 & 449.848 & 30.223 & 3.302 & 0.016 & 1.679 & 914\end{array}$ $\begin{array}{lllllllllllll}45 & -86.59 & 48.00 & 160.736 & 219.19 & 645.194 & 449.848 & 30.223 & 3.302 & 0.016 & 1.679 & 914 & 3\end{array}$ $\begin{array}{llllllllllll}46^{\mathrm{a}} & -485.45 & 229.40236 .856 & 271.82 & 666.641 & 492.438 & 24.124 & 5.250 & 0.007 & 0.876 & 1684 & 5\end{array}$ $\begin{array}{lllllllllllll}47 & -96.38 & 39.45 & 160.802 & 205.23 & 640.795 & 444.099 & 30.390 & 3.253 & 0.020 & 1.549 & 868\end{array}$ $\begin{array}{llllllllllll}48 & -213.60 & -40.77 & 175.231 & 210.31 & 623.846 & 437.529 & 28.719 & 2.653 & 0.029 & 1.823 & 868\end{array}$

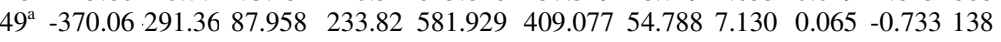
$\begin{array}{lllllllllll}50 & -395.98 & 285.38111 .403 & 230.09 & 577.980 & 410.748 & 48.292 & 7.007 & 0.053 & -0.204 & 224\end{array}$ $\begin{array}{llllllllllll}51 & -317.57 & -86.44 & 180.519 & 285.95 & 684.788 & 467.908 & 29.861 & 2.310 & 0.021 & 2.422 & 878\end{array}$

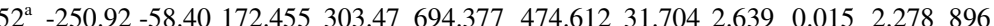
$\begin{array}{llllllllllll}53 & -245.82 & -45.20 & 172.707 & 283.81 & 706.296 & 490.534 & 31.280 & 3.206 & 0.010 & 2.298 & 992\end{array}$ $\begin{array}{lllllllllll}54 & -391.01 & 258.40114 .075 & 205.88 & 697.621 & 464.744 & 40.934 & 2.254 & 0.024 & -0.284 & 328\end{array}$ $\begin{array}{llllllllllll}55 & -350.03 & 259.11 & 88.959 & 198.85 & 689.010 & 450.107 & 48.630 & 2.377 & 0.029 & -0.803 & 212\end{array}$ $\begin{array}{llllllllllll}56 & -84.50 & -0.03 & 129.725 & 255.77 & 684.789 & 462.990 & 39.456 & 3.368 & 0.007 & 1.581 & 726\end{array}$ $\begin{array}{llllllllllll}57 & -411.65 & 249.98 & 137.005 & 217.15 & 712.100 & 484.582 & 36.333 & 2.131 & 0.017 & 0.245 & 496\end{array}$ $\begin{array}{lllllllllll}58^{\mathrm{a}} & -353.87 & 220.02129 .267 & 217.91 & 718.649 & 487.120 & 37.963 & 2.460 & 0.011 & 0.381 & 496\end{array}$ $\begin{array}{lllllllllll}59 & -388.62 & 180.99177 .402 & 371.69 & 756.520 & 526.996 & 32.992 & 6.259 & 0.004 & 1.029 & 1066\end{array}$ $\begin{array}{lllllllllllll}60 & -211.48 & 41.46 & 247.358 & 320.94 & 734.941 & 532.652 & 22.398 & 3.187 & 0.003 & 3.710 & 2400 & 2\end{array}$ $\begin{array}{lllllllllllll}61 & -202.61 & 39.54 & 247.033 & 337.70 & 737.307 & 536.100 & 22.784 & 3.187 & 0.003 & 3.770 & 2400 & 2\end{array}$ $\begin{array}{lllllllllllll}62 & -170.19 & 133.56 & 55.999 & 153.80 & 486.186 & 307.015 & 56.026 & 2.629 & 0.236 & -0.224 & 40 & 0\end{array}$ $\begin{array}{lllllllllllll}63 & -190.83 & 125.14 & 78.930 & 165.07 & 514.311 & 332.961 & 48.766 & 2.507 & 0.167 & 0.305 & 90\end{array}$

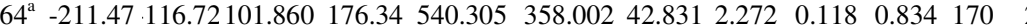

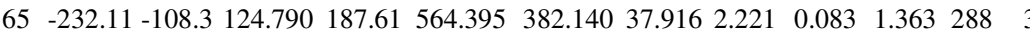
$\begin{array}{lllllllllllll}66 & -252.75 & -99.88 & 147.721 & 198.88 & 586.774 & 405.374 & 33.802 & 2.060 & 0.059 & 1.892 & 452 & 4\end{array}$ $\begin{array}{lllllllllllll}67 & -273.39 & -91.46 & 170.651 & 210.15 & 607.611 & 427.704 & 30.323 & 1.859 & 0.042 & 2.421 & 670 & 5\end{array}$ $\begin{array}{lllllllllllll}68 & -294.03 & -83.04 & 193.582 & 221.42 & 627.054 & 449.130 & 27.354 & 1.678 & 0.029 & 2.950 & 950 & 6\end{array}$ $\begin{array}{lllllllllllll}69 & -314.67 & -74.62 & 216.512 & 232.69 & 645.230 & 469.652 & 24.801 & 1.523 & 0.021 & 3.479 & 1300 & 7\end{array}$ $\begin{array}{lllllllllllll}70 & -335.31 & -66.20 & 239.442 & 243.96 & 662.251 & 489.270 & 22.590 & 1.581 & 0.015 & 4.008 & 1728 & 8\end{array}$ $\begin{array}{lllllllllllll}71^{\mathrm{a}} & -94.25 & -36.50 & 87.430 & 171.26 & 559.755 & 365.533 & 45.902 & 3.066 & 0.079 & 0.520 & 170\end{array}$

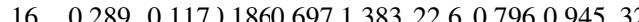

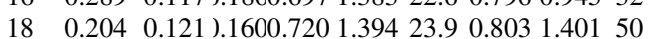
$\begin{array}{lllllllll}20 & 0.144 & 0.124 & ) .1400 .736 & 1.403 & 24.9 & 0.808 & 1.857 & 79\end{array}$ $\begin{array}{llllllllll}22 & 0.102 & 0.126 & 0.1250 .749 & 1.410 & 25.8 & 0.812 & 2.313 & 114\end{array}$ $\begin{array}{lllllllll}18 & 0.289 & 0.096) .1900 .714 & 1.411 & 23.1 & 0.826 & 0.771 & 32\end{array}$ $\begin{array}{lllllllll}24 & 0.102 & 0.112 & \text { ).1270.761 } & 1.422 & 25.8 & 0.825 & 2.608 & 110\end{array}$

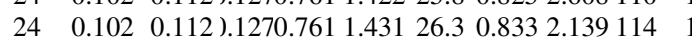
$\begin{array}{lllllllllll}22 & 0.333 & 0.070 & 0.3720 .558 & 1.381 & 26.4 & 0.969 & -1.340 & 29\end{array}$ $\begin{array}{lllllllll}30 & 0.083 & 0.099 & ) .2250 .676 & 1.419 & 29.4 & 0.918 & 0.628 & 143\end{array}$ $\begin{array}{llllllll}26 & 0.102 & 0.097) .1290 .774 & 1.454 & 26.8 & 0.856 & 1.965114\end{array}$

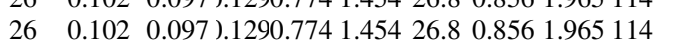
$\begin{array}{lllllllll}34 & 0.056 & 0.107 & 0.1880 .705 & 1.426 & 28.2 & 0.902 & 1.036223\end{array}$ $\begin{array}{llllllll}26 & 0.118 & 0.097) .1290 .774 & 1.452 & 25.7 & 0.857 & 1.493 & 108\end{array}$

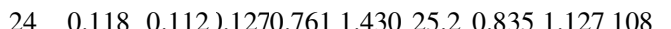

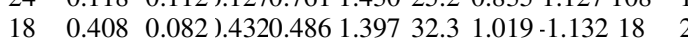
$\begin{array}{llllllllllll}20 & 0.333 & 0.092 & ) .3630 .545 & 1.408 & 30.4 & 0.983 & -0.874 & 29 & 2\end{array}$ $\begin{array}{lllllllll}26 & 0.059 & 0.115) & 1140.771 & 1.431 & 24.8 & 0.869 & 2.259 & 106\end{array}$ $28 \quad 0.059 \quad 0.102) .1160 .7821 .45526 .40 .9051 .865109$ $\begin{array}{llllllllll}28 & 0.042 & 0.102 & \text { ).1160.782 } & 1.469 & 30.9 & 0.931 & 1.773 & 121 & 1\end{array}$ $\begin{array}{lllllllll}24 & 0.118 & 0.081 & 0.3200 .600 & 1.430 & 29.3 & 1.040 & 0.932 & 41\end{array}$ $\begin{array}{llllllllll}22 & 0.144 & 0.070 & 0.3720 .558 & 1.442 & 35.4 & 1.128 & -0.475 & 26\end{array}$ $\begin{array}{lllllll}30 & 0.059 & 0.067) 1330.8001 .511 & 34.1 & 0.993 & 1.625 & 88\end{array}$ $\begin{array}{llllllllll}26 & 0.083 & 0.088 & ) .2800 .631 & 1.431 & 29.0 & 1.002 & 1.388 & 63\end{array}$ $\begin{array}{llllllllll}26 & 0.083 & 0.088 & 1.2800 .631 & 1.431 & 29.0 & 1.002 & 1.388 & 63 \\ 28 & 0.083 & 0.072 & 1.2850 .643 & 1.455 & 30.5 & 1.046 & 1.214 & 63\end{array}$ $\begin{array}{lllllllll}36 & 0.048 & 0.079) .2100 .710 & 1.469 & 30.8 & 1.020 & 0.936138\end{array}$ $\begin{array}{llllllll}38 & 0.017 & 0.105 & 0.0830 .812 & 1.511 & 32.7 & 0.935 & 2.751301\end{array}$ $\begin{array}{llllllll}38 & 0.017 & 0.105 & 0.0830 .812 & 1.517 & 33.6 & 0.944 & 2.597301\end{array}$ $\begin{array}{llllllllll}10 & 0.707 & 0.092 & ) .3630 .545 & 1.314 & 17.6 & 0.748 & 0.054 & 4\end{array}$ $\begin{array}{llllllllll}12 & 0.500 & 0.104 & 5.2750 .620 & 1.347 & 20.5 & 0.770 & 0.590 & 10\end{array}$ $\begin{array}{llllllll}14 & 0.354 & 0.112) .2220 .6661 .369 & 22.5 & 0.784 & 0.966 & 20\end{array}$ $\begin{array}{lllllllll}16 & 0.250 & 0.117) .1860 .6971 .384 & 24.0 & 0.794 & 1.422 & 35\end{array}$ $\begin{array}{llllllllllll}18 & 0.177 & 0.121 & ) .1600 .720 & 1.396 & 25.1 & 0.801 & 1.878 & 56\end{array}$ $\begin{array}{lllllllll}20 & 0.125 & 0.124 & 0.1400 .736 & 1.405 & 26.0 & 0.807 & 2.334 & 84\end{array}$ $\begin{array}{llllllllll}22 & 0.088 & 0.126 & ) .1250 .749 & 1.412 & 26.8 & 0.811 & 2.790 & 120\end{array}$ $\begin{array}{llllllll}24 & 0.063 & 0.128 & 1 & 1120.7601 .417 & 27.4 & 0.815 & 3.246165\end{array}$

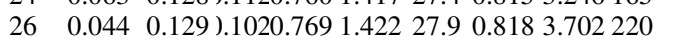

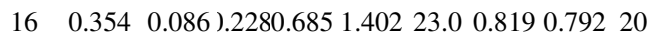

$\begin{array}{ll}0 & 2.6317 .1 \\ 0\end{array}$ $\begin{array}{ll}0 & 2.8317 .1 \\ 0 & 2.7217 .1\end{array}$ $\begin{array}{ll}0 & 2.7217 .1\end{array}$ 0 2.7217 .1 $\begin{array}{ll}0 & 2.6317 .1\end{array}$ $0 \quad 2.8817 .1$ $\begin{array}{ll}0 & 2.7517 .1\end{array}$ $\begin{array}{ll}0 & 2.9934 .1\end{array}$ $\begin{array}{lll}0 & 3.1234 .1\end{array}$ $\begin{array}{ll}0 & 2.7517 .1\end{array}$ $0 \quad 2.7517 .1$ $\begin{array}{ll}0 & 3.6134 .1\end{array}$ $\begin{array}{ll}0 & 2.9117 .1\end{array}$ \begin{tabular}{ll}
0 & 2.9117 .1 \\
\hline &
\end{tabular} $\begin{array}{ll}1 & 2.5437 .3\end{array}$ $1 \quad 2.9937 .3$ 0 2.6017 .1 $\begin{array}{ll}0 & 2.5117 .1\end{array}$ $\begin{array}{ll}0 & 2.2517 .1\end{array}$ $\begin{array}{ll}0 & 1.9326 .3\end{array}$ $\begin{array}{ll}0 & 2.1826 .3\end{array}$ $\begin{array}{ll}0 & 1.9817 .1\end{array}$ o 2.2226 .3 $\begin{array}{ll}0 & 2.2226 .3\end{array}$ $\begin{array}{ll}0 & 2.4334 .1\end{array}$ $\begin{array}{ll}0 & 2.5217 .1\end{array}$ $\begin{array}{ll}0 & 2.5217 .1\end{array}$ $\begin{array}{ll}0 & 1.6317 .1\end{array}$ $\begin{array}{ll}0 & 1.9717 .1\end{array}$ $\begin{array}{ll}0 & 2.1917 .1\end{array}$ $\begin{array}{ll}0 & 2.3417 .1\end{array}$ $\begin{array}{ll}0 & 2.4517 .1\end{array}$ $\begin{array}{ll}0 & 2.5317 .1\end{array}$ $\begin{array}{ll}0 & 2.6017 .1\end{array}$ $\begin{array}{ll}0 & 2.6517 .1\end{array}$ $\begin{array}{ll}0 & 2.6917 .1\end{array}$ o 2.1917 .1 
$\begin{array}{llllllllllll}72 & -114.89 & -28.08 & 110.361 & 182.53 & 582.954 & 389.393 & 40.466 & 2.781 & 0.056 & 1.049 & 288\end{array}$ $\begin{array}{llllllllllll}73 & -135.53 & -19.66 & 133.291 & 193.8 & 604.447 & 412.348 & 35.942 & 2.699 & 0.039 & 1.578 & 452\end{array}$ $\begin{array}{lllllllllllll}74 & -156.17 & -11.24 & 156.221 & 205.07 & 624.405 & 434.400 & 32.137 & 2.541 & 0.028 & 2.107 & 670\end{array}$ $\begin{array}{lllllllllllll}75 & -156.17 & -11.24 & 156.221 & 205.07 & 624.405 & 434.400 & 32.137 & 2.541 & 0.028 & 1.937 & 670 & 4\end{array}$ $\begin{array}{lllllllllllll}76^{\mathrm{a}} & -176.81 & -2.82 & 179.152 & 216.34 & 642.975 & 455.547 & 28.905 & 2.523 & 0.020 & 2.636 & 950 & 5\end{array}$ $\begin{array}{lllllllllllll}77^{\mathrm{a}} & -38.95 & 68.98 & 141.792 & 199.99 & 641.743 & 441.010 & 34.119 & 3.141 & 0.019 & 1.683 & 670\end{array}$ $\begin{array}{llllllllllllll}78^{\mathrm{a}} & -38.95 & 68.98 & 141.792 & 199.99 & 641.743 & 441.010 & 34.119 & 3.141 & 0.019 & 1.683 & 670 & 3\end{array}$ $\begin{array}{lllllllllllll}79 & -80.23 & 85.82 & 187.652 & 222.53 & 675.804 & 481.844 & 27.585 & 2.901 & 0.009 & 2.681 & 1300\end{array}$ $\begin{array}{lllllllllll}80 & -237.39 & 110.74125 .305 & 172.61 & 547.521 & 367.698 & 38.293 & 2.145 & 0.096 & 1.363 & 260\end{array}$ $\begin{array}{lllllllllll}81 & -237.39 & 110.74125 .305 & 172.61 & 547.521 & 367.698 & 38.293 & 2.132 & 0.096 & 1.233 & 266\end{array}$ $\begin{array}{lllllllllllll}82^{\mathrm{a}} & -95.83 & -37.43 & 91.383 & 160.62 & 536.918 & 349.399 & 45.592 & 2.852 & 0.083 & 0.300 & 156\end{array}$ $\begin{array}{lllllllllllll}83^{\mathrm{a}} & -124.68 & -36.63 & 110.427 & 168.57 & 577.373 & 382.971 & 40.725 & 2.745 & 0.068 & 0.829 & 260\end{array}$ $\begin{array}{lllllllllllll}84 & -145.32 & -28.21 & 133.357 & 179.84 & 599.295 & 406.173 & 36.159 & 2.625 & 0.048 & 1.358 & 410\end{array}$ $\begin{array}{lllllllllllll}85 & -36.86 & 20.95 & 110.781 & 236.57 & 683.038 & 454.392 & 45.347 & 2.959 & 0.008 & 1.495 & 530\end{array}$ $\begin{array}{llllllllllll}86^{\mathrm{a}} & -89.61 & 28.16 & 158.503 & 271.63 & 711.743 & 494.068 & 34.930 & 2.684 & 0.005 & 2.523 & 1008\end{array}$ $\begin{array}{lllllllllllll}87^{\mathrm{a}} & -110.25 & 36.58 & 181.433 & 282.90 & 724.923 & 512.555 & 31.280 & 2.564 & 0.003 & 3.052 & 1360 & 3\end{array}$ $\begin{array}{llllllllllll}88 & -230.91 & -36.54 & 196.658 & 301.04 & 694.045 & 486.905 & 29.282 & 2.666 & 0.010 & 3.350 & 1138\end{array}$ $\begin{array}{llllllllllll}89 & -173.13 & -6.58 & 188.920 & 301.80 & 700.094 & 489.428 & 30.457 & 3.266 & 0.007 & 2.926 & 1138\end{array}$ $\begin{array}{llllllllllll}90 & -298.76 & 145.60133 .448 & 206.43 & 573.422 & 410.132 & 38.771 & 3.275 & 0.112 & 1.352 & 288 & 3\end{array}$

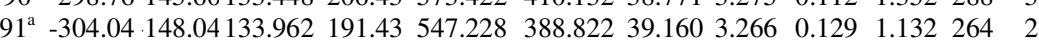
$\begin{array}{lllllllllllll}92 & -319.40 & 137.18 & 156.378 & 217.70 & 594.658 & 432.273 & 34.521 & 3.113 & 0.079 & 1.881 & 452 & 4\end{array}$ $\begin{array}{llllllllllll}93 & -360.68 & 120.34202 .239 & 240.24 & 633.340 & 473.841 & 27.877 & 3.002 & 0.040 & 2.939 & 950 & 6\end{array}$ $\begin{array}{llllllllllll}94 & -178.61 & -60.20 & 123.419 & 189.67 & 549.256 & 387.069 & 41.303 & 3.775 & 0.075 & 0.848 & 264\end{array}$ $\begin{array}{llllllllllll}95 & -181.54 & -65.38 & 119.018 & 201.35 & 589.514 & 417.048 & 41.409 & 3.811 & 0.075 & 1.068 & 288\end{array}$ $\begin{array}{llllllllllll}96^{\mathrm{a}} & -181.54 & -65.38 & 119.018 & 201.35 & 589.514 & 417.048 & 41.409 & 3.811 & 0.075 & 1.068 & 288\end{array}$ $\begin{array}{llllllllllll}97 & -240.53 & -34.94 & 192.210 & 223.48 & 613.269 & 453.492 & 29.409 & 3.415 & 0.026 & 2.435 & 890\end{array}$ $\begin{array}{llllllllllll}98 & -243.46 & -40.12 & 187.809 & 235.16 & 647.030 & 479.922 & 29.473 & 3.451 & 0.026 & 2.655 & 950\end{array}$ $\begin{array}{lllllllllll}99 & -440.91 & 295.72124 .561 & 225.98 & 579.345 & 429.939 & 50.875 & 5.026 & 0.067 & -0.751 & 224\end{array}$ $\begin{array}{lllllllllll}100^{\mathrm{a}}-123.31 & 45.28 & 177.780 & 218.40 & 627.656 & 459.851 & 31.140 & 4.015 & 0.018 & 1.951 & 890\end{array}$ $\begin{array}{llllllllllll}101 & -115.10 & 52.90 & 181.666 & 221.72 & 615.302 & 451.936 & 30.796 & 4.015 & 0.015 & 1.951 & 890\end{array}$

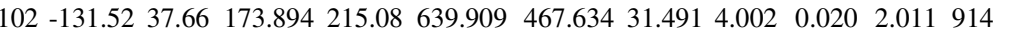
$\begin{array}{lllllllllll}103 & -329.96 & 142.06157 .407 & 187.70 & 554.429 & 398.057 & 35.179 & 2.837 & 0.105 & 1.531 & 386\end{array}$ $\begin{array}{lllllllllll}104^{\mathrm{a}}-365.96 & 122.78202 .753 & 225.24 & 620.319 & 461.693 & 28.114 & 2.897 & 0.046 & 2.809 & 838\end{array}$ $\begin{array}{lllllllllll}105 & -365.96 & 122.78202 .753 & 225.24 & 620.319 & 461.693 & 28.114 & 2.897 & 0.046 & 2.809 & 838\end{array}$ $\begin{array}{lllllllllll}106 & -615.04 & 343.64210 .480 & 243.43 & 629.885 & 471.289 & 30.323 & 7.329 & 0.012 & -0.1641016 & 5\end{array}$ $\begin{array}{lllllllllll}107 & -615.04 & 343.64210 .480 & 243.43 & 621.182 & 464.778 & 30.323 & 7.329 & 0.012 & -0.1641054 & 5\end{array}$ $\begin{array}{lllllll}18 & 0.250 & 0.096) 1900.7141 .413 & 24.6 & 0.8241 .248 \quad 35\end{array}$ $\begin{array}{lllllllllll}20 & 0.177 & 0.103 & 1630.734 & 1.421 & 25.7 & 0.828 & 1.704 & 56\end{array}$

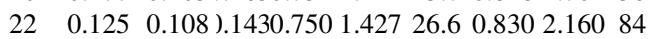
$\begin{array}{llllllllll}22 & 0.125 & 0.108 & \text { ).1430.750 } 1.427 & 26.6 & 0.830 & 1.620 & 84\end{array}$ $\begin{array}{llllllll}24 & 0.088 & 0.112 & \text { ).1270.761 } & 1.432 & 27.3 & 0.832 & 2.616120\end{array}$ $\begin{array}{lllllllll}24 & 0.125 & 0.092) & 1450.763 & 1.453 & 27.2 & 0.856 & 1.986 & 84\end{array}$

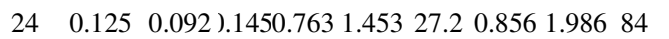
$\begin{array}{lllllllll}28 & 0.063 & 0.102 & ) .1160 .782 & 1.457 & 28.5 & 0.855 & 2.358 & 165\end{array}$ $\begin{array}{llllllllll}16 & 0.289 & 0.117) .1860 .697 & 1.382 & 22.6 & 0.791 & 1.170 & 31\end{array}$ $\begin{array}{llllllllll}16 & 0.289 & 0.117 & ) .1860 .697 & 1.382 & 22.6 & 0.791 & 1.170 & 32\end{array}$ $\begin{array}{lllllllll}16 & 0.408 & 0.086) .2280 .685 & 1.384 & 20.8 & 0.807 & 0.789 & 18\end{array}$ $\begin{array}{llllllllll}18 & 0.289 & 0.096) .1900 .714 & 1.411 & 23.1 & 0.826 & 0.776 & 31\end{array}$ $\begin{array}{lllllllllll}20 & 0.204 & 0.103 & \text { ).1630.734 } 1.419 & 24.4 & 0.829 & 1.232 & 50\end{array}$ $\begin{array}{llllllll}28 & 0.072 & 0.057) .1510 .792 & 1.566 & 38.8 & 1.049 & 1.455 & 64\end{array}$ $\begin{array}{llllllll}32 & 0.042 & 0.075) .1190 .806 & 1.548 & 36.3 & 1.001 & 2.214 & 125\end{array}$

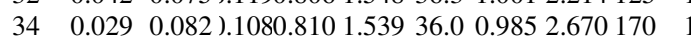

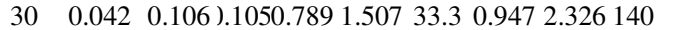
$\begin{array}{llllllll}32 & 0.042 & 0.094) .1070 .8001 .529 & 34.7 & 0.975 & 1.822 & 140\end{array}$ $\begin{array}{lllllllll}14 & 0.250 & 0.137) .1810 .681 & 1.407 & 27.1 & 0.811 & 1.480 & 35\end{array}$ $\begin{array}{lllllllll}14 & 0.289 & 0.137 & ) .1810 .681 & 1.405 & 25.6 & 0.809 & 1.210 & 31\end{array}$ $\begin{array}{lllllllll}16 & 0.177 & 0.138 & 0.1570 .705 & 1.415 & 27.9 & 0.8162 .025 & 56\end{array}$ $\begin{array}{lllllllll}20 & 0.088 & 0.139 & ) .1230 .738 & 1.427 & 29.0 & 0.823 & 3.150 & 120\end{array}$ $\begin{array}{lllllllll}16 & 0.289 & 0.1177) .1860 .6971 .421 & 25.6 & 0.827 & 1.022 & 31\end{array}$ $\begin{array}{lllllllll}16 & 0.250 & 0.117 & ) .1860 .697 & 1.437 & 27.9 & 0.842 & 0.751 & 35\end{array}$ $\begin{array}{llllllllll}16 & 0.250 & 0.117 & 1.1860 .697 & 1.437 & 27.9 & 0.842 & 0.751 & 35\end{array}$ $\begin{array}{llllllll}22 & 0.102 & 0.126) .1250 .749 & 1.438 & 28.0 & 0.834 & 2390110\end{array}$ $\begin{array}{llllllll}22 & 0.088 & 0.126) .1250 .749 & 1.449 & 29.7 & 0.845 & 2.119 & 120\end{array}$ $\begin{array}{llllllllll}18 & 0.333 & 0.112 & ) .3550 .533 & 1.434 & 34.8 & 0.997 & -0.920 & 29 & 2\end{array}$ $\begin{array}{lllllllll}24 & 0.102 & 0.112 & ) .1270 .761 & 1.461 & 28.7 & 0.857 & 1.676 & 110\end{array}$ $\begin{array}{llllllllll}24 & 0.102 & 0.112) .1270 .761 & 1.451 & 28.1 & 0.848 & 2.145110\end{array}$

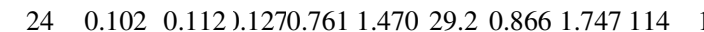
$\begin{array}{lllllllll}16 & 0.236 & 0.138 & ) .1570 .705 & 1.411 & 25.3 & 0.811 & 1.471 & 46\end{array}$ $\begin{array}{llllllllll}20 & 0.102 & 0.139 & ) .1230 .738 & 1.426 & 28.0 & 0.821 & 2.581 & 104 & 1\end{array}$ $\begin{array}{llllllllll}20 & 0.102 & 0.139 & ) .1230 .738 & 1.426 & 28.0 & 0.821 & 2.581 & 104\end{array}$ $\begin{array}{llllllllll}30 & 0.083 & 0.109) .3240 .567 & 1.422 & 29.5 & 0.955 & 0.053 & 141 & 3\end{array}$

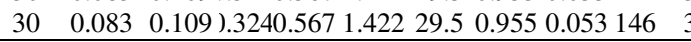




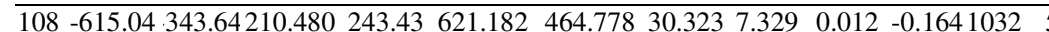

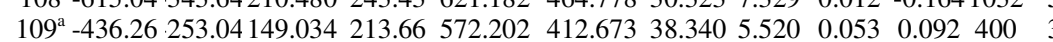
$\begin{array}{lllllllllll}110^{\mathrm{a}}-318.68 & 120.09156 .535 & 240.58 & 675.486 & 479.865 & 37.226 & 3.452 & 0.023 & 2.385 & 726 & 0\end{array}$ $\begin{array}{lllllllllll}111^{\mathrm{a}}-311.46 & 109.09165 .657 & 267.28 & 632.221 & 450.116 & 35.558 & 3.452 & 0.032 & 2.305 & 670 & 0\end{array}$ $\begin{array}{llllllllllll}112 & -103.51 & -16.35 & 119.438 & 255.39 & 679.076 & 478.828 & 46.466 & 5.377 & 0.010 & 1.104 & 530\end{array}$ $\begin{array}{llllllllllll}113^{\mathrm{a}}-434.88 & 377.95 & 65.634 & 272.40 & 594.481 & 395.480 & 57.305 & 4.720 & 0.091 & -0.194 & 72 & 0\end{array}$ $\begin{array}{llllllllllll}114^{\mathrm{a}}-476.16 & 361.11111 .495 & 294.94 & 634.708 & 440.014 & 43.684 & 5.004 & 0.046 & 0.864 & 244 & 2\end{array}$ $\begin{array}{llllllllllll}115 & -517.44 & 344.27157 .356 & 317.48 & 669.451 & 480.932 & 34.399 & 4.422 & 0.023 & 1.922 & 592 & 4\end{array}$ $\begin{array}{llllllllllll}116 & -538.08 & 335.85180 .286 & 328.75 & 685.075 & 500.036 & 30.830 & 4.302 & 0.016 & 2.451 & 852\end{array}$ $\begin{array}{llllllllllll}117 & -330.09 & 281.69 & 78.021 & 281.91 & 618.906 & 416.532 & 52.893 & 5.382 & 0.037 & 0.351 & 140\end{array}$ $\begin{array}{llllllllllll}118 & -481.44 & 363.55112 .009 & 279.94 & 620.536 & 426.972 & 44.150 & 4.619 & 0.053 & 0.644 & 224\end{array}$ $\begin{array}{lllllllllllll}119 & -502.08 & 355.13134 .940 & 291.21 & 639.447 & 448.428 & 39.014 & 4.493 & 0.037 & 1.263 & 364 & 2\end{array}$ $120^{\mathrm{a}}-621.32 \begin{array}{lllllllllll}458.41 & 149.875 & 233.76 & 602.937 & 439.370 & 39.407 & 6.335 & 0.015 & 0.331 & 504 & 3\end{array}$ $\begin{array}{lllllllllllll}121 & 12.90 & 15.66 & 45.881 & 158.59 & 558.579 & 337.232 & 56.026 & 0.659 & 1.435 & 1.466 & 10\end{array}$ $\begin{array}{lllllllllllll}122^{\mathrm{a}} & -7.74 & 24.08 & 68.811 & 169.86 & 583.293 & 362.122 & 48.766 & 0.549 & 1.015 & 1.995 & 36\end{array}$ $\begin{array}{llllllllllllll}123 & -33.66 & 30.06 & 92.256 & 166.13 & 589.194 & 371.757 & 43.283 & 0.329 & 0.828 & 2.524 & 78\end{array}$ $\begin{array}{lllllllllllll}124 & -69.66 & 49.34 & 137.603 & 203.67 & 645.943 & 431.368 & 33.802 & 0.161 & 0.359 & 3.582 & 272 & 3\end{array}$ $\begin{array}{llllllllllll}125 & -131.58 & 74.60 & 206.394 & 237.48 & 695.229 & 492.478 & 24.801 & -0.199 & 0.127 & 5.169 & 913\end{array}$ $\begin{array}{lllllllllllll}126 & -36.84 & 67.13 & 123.262 & 173.77 & 578.087 & 383.761 & 34.889 & 0.441 & 0.164 & 2.568 & 272 & 3\end{array}$ $\begin{array}{lllllllllllll}127^{\mathrm{a}} & -42.74 & -0.92 & 72.393 & 146.20 & 508.311 & 315.706 & 51.534 & 1.138 & 1.225 & 0.842 & 40\end{array}$ $\begin{array}{lllllllllllll}128 & -93.62 & -0.35 & 90.428 & 201.36 & 586.184 & 375.543 & 48.427 & 3.574 & 0.039 & 1.817 & 236\end{array}$ $\begin{array}{lllllllllllll}129 & -114.26 & 8.07 & 113.358 & 212.63 & 608.120 & 399.028 & 42.552 & 3.467 & 0.028 & 2.346 & 374\end{array}$ $\begin{array}{llllllllllll}130^{\mathrm{a}}-176.18 & 33.33 & 182.149 & 246.44 & 664.456 & 464.057 & 30.156 & 3.107 & 0.010 & 3.933 & 1144 & 4\end{array}$ $\begin{array}{lllllllllllll}131 & -58.96 & 113.55 & 167.719 & 241.36 & 681.556 & 470.273 & 31.955 & 3.707 & 0.007 & 3.449 & 1144 & 3\end{array}$ $\begin{array}{llllllllllllll}132 & 123.47 & 256.52 & 124.102 & 338.90 & 666.487 & 441.819 & 45.902 & 3.837 & 0.017 & 0.686 & 520 & 0\end{array}$ $\begin{array}{llllllllllllll}133^{\mathrm{a}} & 59.25 & 254.10 & 173.685 & 386.48 & 696.374 & 482.568 & 34.684 & 3.752 & 0.013 & 1.584 & 926 & 0\end{array}$ $\begin{array}{lllllllllllll}134^{\mathrm{a}} & 81.51 & 197.52 & 125.797 & 266.11 & 652.541 & 431.753 & 41.197 & 3.171 & 0.018 & 1.673 & 560\end{array}$ $\begin{array}{lllllllllllll}135 & -37.72 & 85.16 & 91.774 & 155.54 & 457.959 & 292.005 & 43.974 & 2.469 & 0.447 & 0.018 & 90\end{array}$ $\begin{array}{llllllllllll}136^{\mathrm{a}} & -259.49 & -76.99 & 162.386 & 263.43 & 621.800 & 424.687 & 32.430 & 2.433 & 0.029 & 1.743 & 696\end{array}$ $\begin{array}{lllllllllllll}137 & -219.95 & -1.30 & 200.399 & 247.37 & 663.196 & 464.132 & 27.498 & 2.280 & 0.010 & 3.082 & 1314 & 2\end{array}$

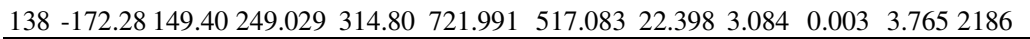

$\begin{array}{llllllll}0 & 30 & 0.083 & 0.109) .3240 .5671 .422 & 29.5 & 0.9550 .053143 & 3\end{array}$ $\begin{array}{llllllllll}20 & 0.204 & 0.116 & ) .3070 .577 & 1.408 & 27.8 & 0.903 & 0.273 & 52\end{array}$ $\begin{array}{lllllllll}22 & 0.051 & 0.126 & 0.1250 .749 & 1.466 & 30.7 & 0.922 & 2.285 & 88\end{array}$

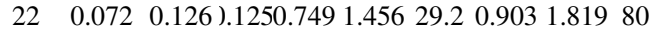

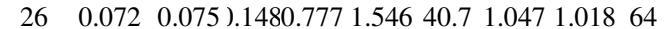

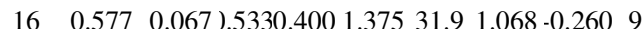
$\begin{array}{lllllllll}20 & 0.289 & 0.092 & 0.3630 .545 & 1.411 & 32.5 & 0.987 & 0.885 & 32\end{array}$ $\begin{array}{lllllllll}24 & 0.144 & 0.104) .2750 .620 & 1.427 & 32.8 & 0.950 & 1.912 & 79\end{array}$

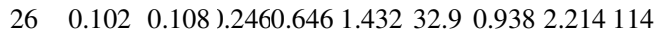
$\begin{array}{llllllll}20 & 0.408 & 0.056) .4440 .500 & 1.422 & 32.8 & 1.063 & 0.685 & 18\end{array}$ $\begin{array}{lllllllll}20 & 0.333 & 0.092 & ) .3630 .545 & 1.408 & 30.4 & 0.983 & 0.594 & 29\end{array}$

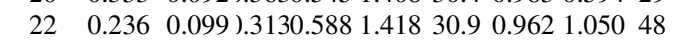
$\begin{array}{llllllllllll}0 & 28 & 0.167 & 0.085 & 0.4060 .508 & 1.420 & 33.2 & 1.050 & -0.217 & 70\end{array}$ $\begin{array}{lllllllllllll}0 & 1.486 & 1.000 & 0.021 & 0 & 0.085 & 1.527 & 27.2 & 2.222 & 1.600 & 1 & 0\end{array}$ $\begin{array}{lllllllllllll}1 & 3.486 & 0.707 & 0.032 & 0 & 0.154 & 1.516 & 28.5 & 1.940 & 2.000 & 4\end{array}$ $\begin{array}{lllllllllllll}1 & 5.486 & 0.577 & 0.042 & 0 & 0.212 & 1.505 & 27.5 & 1.747 & 2.093 & 9\end{array}$ $\begin{array}{lllllllllllll}0 & 9.486 & 0.250 & 0.056 & 0 & 0.303 & 1.499 & 30.4 & 1.5243 .348 & 35 & 0\end{array}$ $\begin{array}{lllllllllll}1 & 5.486 & 0.088 & 0.071 & 0 & 0.400 & 1.492 & 31.3 & 1.338 & 4.716120 & 0\end{array}$ $\begin{array}{lllllllllllll}0 & 1 & 1.778 & 0.250 & 0.087 & 0 & 0.574 & 1.420 & 23.3 & 0.889 & 2.519 & 35 & 0\end{array}$ $\begin{array}{llllllllllll}1 & 2.667 & 0.707 & 0.097 & 0 & 0.387 & 1.425 & 21.3 & 0.822 & 0.810 & 4\end{array}$ $\begin{array}{lllllllllll}0 & 20 & 0.144 & 0.074 & 0.1950 .731 & 1.441 & 25.5 & 0.927 & 0.930 & 26\end{array}$ $\begin{array}{llllllllllll}1 & 22 & 0.102 & 0.084 & ) .1660 .750 & 1.447 & 26.2 & 0.915 & 2.400 & 43\end{array}$

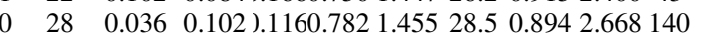
$\begin{array}{lllllllllll}0 & 30 & 0.036 & 0.089 & ) .1170 .794 & 1.479 & 29.1 & 0.919 & 2.349 & 140 & 1\end{array}$ $\begin{array}{lllllllllllll}0 & 26 & 0.083 & 0.075 & 0 & 0.666 & 1.503 & 38.9 & 0.997 & 0.753 & 61 & 2\end{array}$ $\begin{array}{llllllllll}30 & 0.056 & 0.089 & 0 & 0.706 & 1.503 & 36.3 & 0.966 & 1.864 & 111\end{array}$ $\begin{array}{lllllllllll}24 & 0.072 & 0.085 & 0 & 0.785 & 1.498 & 33.6 & 0.927 & 1.859 & 64\end{array}$ $\begin{array}{llllllllllll}8 & 0.577 & 0.153 & 0 & 0.610 & 1.378 & 18.6 & 0.692 & 0.270 & 9\end{array}$ $\begin{array}{llllllllll}24 & 0.083 & 0.112 & 1.1270 .761 & 1.438 & 26.8 & 0.897 & 1.803 & 84\end{array}$ $\begin{array}{lllllllllllll}0 & 30 & 0.034 & 0.106 & 0.1050 .789 & 1.474 & 27.8 & 0.900 & 2.307 & 162\end{array}$ $\begin{array}{lllllllll}38 & 0.010 & 0.105 & ) .0830 .812 & 1.526 & 31.7 & 0.963 & 2.943 & 267\end{array}$

年

${ }^{\mathrm{a}}$ Test set 
The stepwise multiple linear regression procedure based on the forward-selection and backward elimination methods (including the critical probability: $p$-value $<0.05$ for all descriptors and for the model complete), were employed to determine the best regression models. These methods used the coefficients $r, r^{2}$, MSE, F and the $p$-value to select the best regression performance. Where $r$ is the correlation coefficient; $r^{2}$ is the coefficient of determination; MSE is the mean squared error; $\mathrm{F}$ is the fisher $F$-statistic; $p$-value the significance level.

The VIF (Variance inflation factor) was defined ${ }^{13}$ as $1 /\left(1-r^{2}\right)$, where $r$ was the multiple correlation coefficient for an independent variable against all other descriptors in the model. The models with a VIF greater than 5 were unstable and were eliminated, the models with VIF values between 1 and 4 may be accepted (Table 3 ).

Table 3. Multicollinearity statistics

\begin{tabular}{ccccc}
\hline & CT & PC & C\% & NHD \\
\hline VIF & 1.042 & 1.540 & 1.424 & 1.171 \\
\hline
\end{tabular}

The relationship obtained using this method corresponds to the linear combination of these descriptors (Table 3): critical temperature (CT), partition coefficient (PC), percent ratios of carbon $(\mathrm{C} \%)$ and Number of H-Bond donors (NHD). The QSRR models built using stepwise multiple linear regression methods are represented by the following equation:

$$
\begin{aligned}
\log (\mathrm{LRI}) & =2.025+1.611 \times 10^{-3} \times \mathrm{CT}-1.259 \times 10^{-2} \mathrm{X} \mathrm{PC}+0.127 \times \mathrm{C} \%+8.871 \times 10^{-2} \times \mathrm{NHD} \\
\mathrm{N} & =97 ; \mathrm{r}=0.900 ; \mathrm{r}^{2}=0.810 ; \mathrm{MSE}=0.003 ; \mathrm{F}=98.053 ; \mathrm{p} \text {-value }<0.0001 .
\end{aligned}
$$

In this equation, $\mathrm{N}$ is the number of compounds, $\mathrm{r}$ is the correlation coefficient, $\mathrm{r}^{2}$ is the coefficient of determination, MSE is the mean squared error, $\mathrm{F}$ is the Fisher's criterion and $\mathrm{p}$-value is the significance level.

It is observed that the coefficient of correlation $r$ is high and the MSE is low, which makes it's possible to indicate that the model is more reliable. A $p$-value much smaller than 0.05 indicates that the regression equation is statistically significant, we can conclude, with confidence, that the model provides a significant amount of information ${ }^{11}$.

The predicted $\log (\mathrm{LRI})$ values calculated from Eq. 1 are given in Table 4 in comparison to the observed values.

The correlation between the predicted and observed $\log (\mathrm{LRI})$ is shown in Figure 1. The descriptors proposed by MLR are therefore used as input parameters in the MNLR and ANN ${ }^{14,15}$.

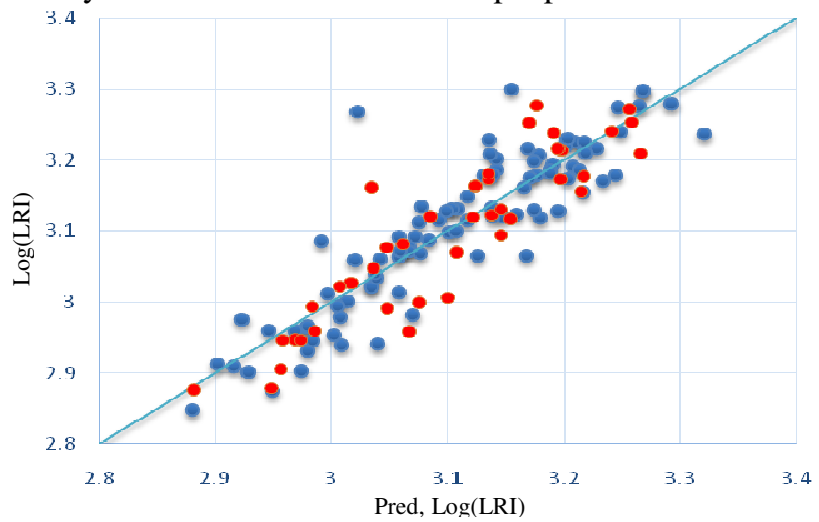

Figure 1. Graphical representation of predicted and observed linear retention indices $(\log (\mathrm{LRI}))$ calculated by MLR (training set in blue; test set in red) 


\section{Multiple non-linear regression (MNLR)}

We used MNLR technique to improve the QSRR by accounting for several parameters. MNLR is the most commonly used tool for the study of multidimensional data. We applied it to the data matrix constituted from the descriptors proposed by the MLR corresponding to the set of 97 molecules $^{16}$. The resulting equation is as follows:

$$
\begin{gathered}
\log (\mathrm{LRI})=1.277+4.240 \times 10^{-3} \times \mathrm{CT}-2.111 \times 10^{-6} \times \mathrm{CT}^{2}-1.080 \times 10^{-2} \times \mathrm{PC}-5.185 \times 10^{-6} \times \\
\operatorname{Exp}(\mathrm{PC})+5.156 \times 10^{-2} \times \mathrm{C} \%+6.452 \times 10^{-2} \times \log (\mathrm{C} \%)+8.142 \times 10^{-2} \times \mathrm{NHD} \\
\mathrm{N}=37 ; \mathrm{r}=0.908 ; \mathrm{r}^{2}=0.824 ; \mathrm{MSE}=0.002 ; \mathrm{F}=59.452 ; \mathrm{p} \text {-value }<0.0001
\end{gathered}
$$

The predicted $\log (\mathrm{LRI})$ values calculated from Eq. 2 are given in Table 4 in comparison to the observed values. The correlation between the predicted and observed $\log (\mathrm{LRI})$ is shown in Figure 2.

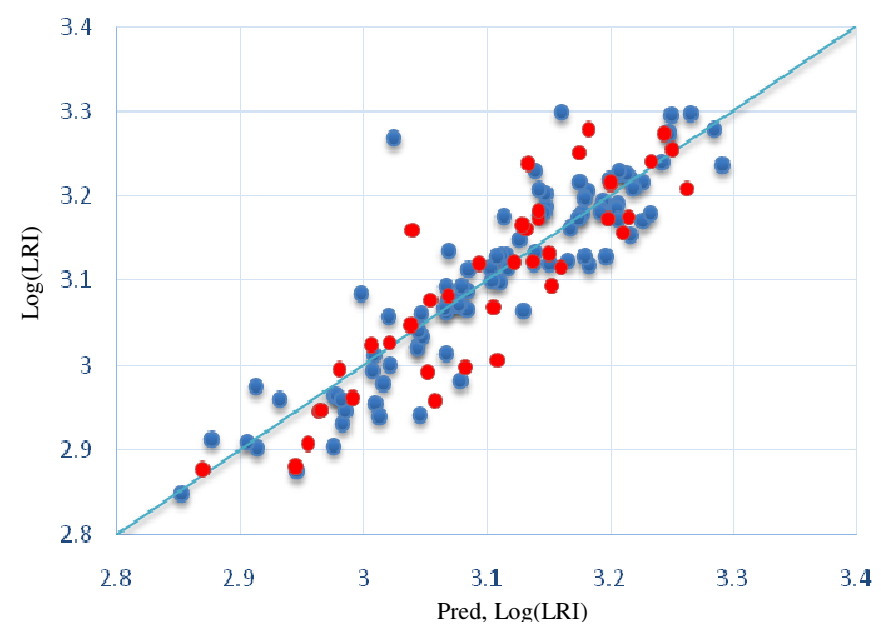

Figure 2. Predicted and observed linear retention indices $(\log (\mathrm{LRI}))$ using MNLR (training set in blue; test set in red)

\section{Artificial neural networks (ANN)}

In order to increase the probability of a good characterization of the molecules studied, the ANN can generate a predictive model of the QSRR relationship between the descriptors obtained from the MLR and the observed linear retention indices.

The ANN model has aroused great interests as its universal function approximators are capable of mapping any linear or nonlinear functions. The multi-layer perceptron (MLP) neuronal network model is a supervised neural network based on the original simple perceptron model with back propagation for training the network. It commonly consists of an input layer of source nodes, an output layer and one or more hidden layers of computation nodes (neurons) that increasing the learning power of the MLP model. The number of hidden neurons determines the learning capacity of MLP network. It is most recommended to select the network which performs best with the least possible number of hidden neurons ${ }^{17}$.

The MLP model was developed using the properties of several molecules studied (Figure 3). The correlation between the predicted and observed $\log (\mathrm{LRI})$ is shown Figure 4. The predicted $\log (\mathrm{LRI})$ values calculated by ANN method are given in Table 4 to comparison to the observed values. 


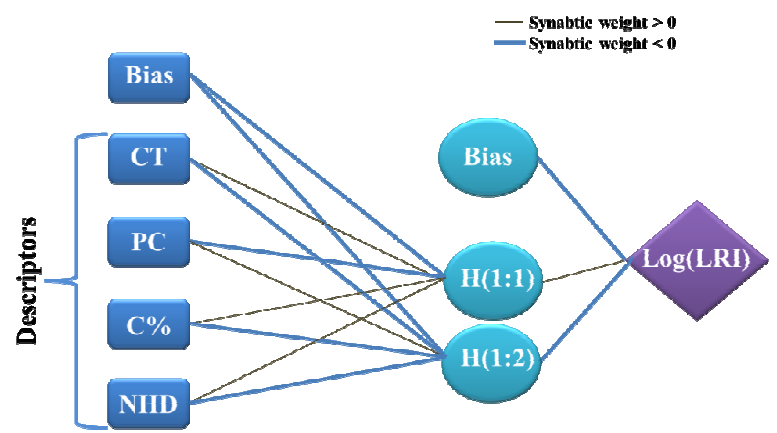

Figure 3. The architecture of the ANN method used (four input variables, two neurons in the hidden layer and one neuron to the output layer)

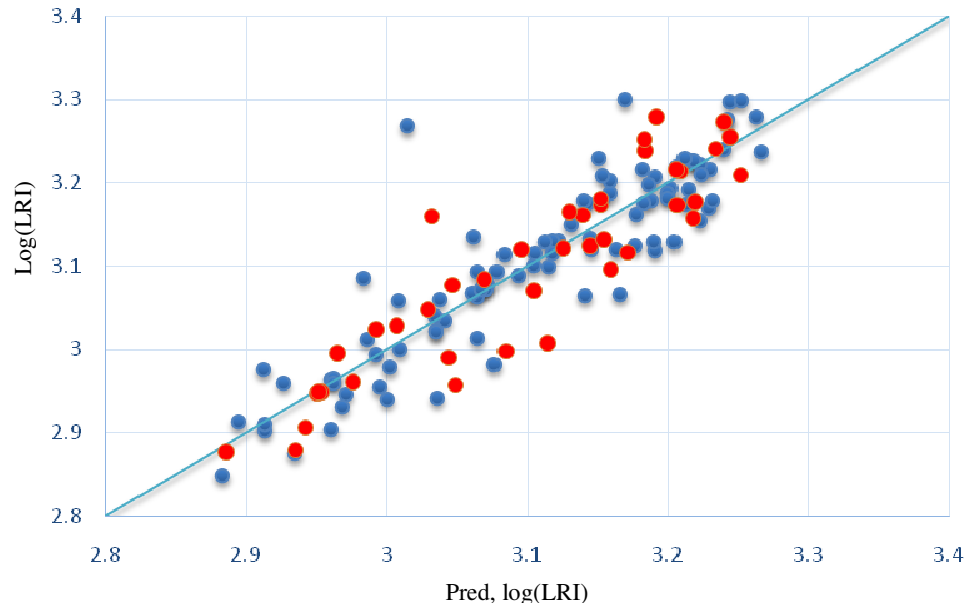

Figure 4. Observed and predicted linear retention indices using ANN (training set in blue; test set in red)

Table 4. Comparison of the observed values with those calculated by MLR, MNLR and ANN methods.

\begin{tabular}{|c|c|c|c|c|c|c|c|c|c|c|c|}
\hline \multirow{2}{*}{$\mathrm{N}^{\circ}$} & \multirow{2}{*}{$\begin{array}{l}\log (\mathrm{LRI}) \\
\quad \text { (obs.) }\end{array}$} & \multicolumn{3}{|c|}{$\begin{array}{c}\log (\text { LRI) } \\
\text { (calc.) }\end{array}$} & \multirow{2}{*}{$\begin{array}{c}\log (\text { LRI }) \\
\text { (obs.) }\end{array}$} & \multicolumn{3}{|c|}{$\begin{array}{c}\log (\text { LRI) } \\
\text { (calc.) }\end{array}$} & \multirow{2}{*}{$\mathrm{N}^{\circ}$} & \multirow{2}{*}{$\begin{array}{l}\log (\mathrm{LRI}) \\
\quad \text { (obs.) }\end{array}$} & \multirow{2}{*}{$\begin{array}{c}\log (\mathrm{LRI}) \\
\frac{\text { (calc.) }}{\text { MLR MNLR ANN }}\end{array}$} \\
\hline & & $\overline{\text { MLR }}$ & MNLR & $\overline{\mathrm{ANN}}$ & & $\overline{\mathrm{MLF}}$ & MNLR & ANN & & & \\
\hline 1 & 2.904 & 2.974 & 2.976 & 2.96047 & 3.208 & 3.136 & 3.143 & 3.153 & 93 & 3.194 & $3.191 \quad 3.194 \quad 3.201$ \\
\hline 2 & & 3.003 & 3.010 & & & & & & 94 & & $\begin{array}{ll}76 & 3.067 \quad 3 .\end{array}$ \\
\hline 3 & & & & $14349^{\mathrm{a}}$ & & & & & 5 & & $139 \quad 3.138 \quad 3.14$ \\
\hline 4 & & & 3.084 & & & & & & $96^{\mathrm{a}}$ & & $\begin{array}{lll}39 & 3.138 & 3.14\end{array}$ \\
\hline 5 & & 949 & 2.946 & & & 96 & 3.196 & & 97 & & 3.1663 .1693 .178 \\
\hline $6^{\mathrm{a}}$ & 39 & 2.949 & 2.946 & $2.93552^{\mathrm{a}}$ & & 214 & 3.212 & 3.217 & 98 & 210 & $\begin{array}{lll}3.218 & 3.219 & 3.22\end{array}$ \\
\hline 7 & 931 & 2.980 & 2.984 & 2.96853 & & 233 & 3.227 & 3.229 & 99 & & $\begin{array}{lll}3.213 & 3.205 & 3.200\end{array}$ \\
\hline 8 & & 3.008 & 3.016 & 3.00254 & & 3.229 & 3.227 & 3.229 & $100^{\mathrm{a}}$ & & $\begin{array}{lll}3.197 & 3.198 & 3.207\end{array}$ \\
\hline 9 & & .034 & 044 & & & & 3.217 & & 101 & & $\begin{array}{lll}3.177 & 3.178 & 3.185\end{array}$ \\
\hline 10 & & & & & & & & & & & $\begin{array}{lll}3.216 & 3.217 & 3.2\end{array}$ \\
\hline 11 & & 059 & 3.067 & & & 249 & 3.2 & 3. & & & $3.077 \quad 3.070 \quad 3.0$ \\
\hline 12 & & 3.059 & 3.067 & $3.06558^{\mathrm{a}}$ & & 3.260 & 3.250 & & & & 3.1753 .18 \\
\hline
\end{tabular}




\begin{tabular}{|c|c|c|c|c|c|c|c|c|c|c|c|c|c|c|}
\hline 13 & 014 & 059 & 067 & 3.065 & 59 & 3.237 & 321 & .292 & 3.266 & 105 & 3.175 & 3.171 & & \\
\hline 14 & & 923 & 914 & 2.913 & 60 & 3.276 & & & & & & & & \\
\hline & & & 955 & 942 & 61 & 6 & 269 & 0 & 3.2 & 77 & & & & \\
\hline & & & & & 62 & & & & & & & & & \\
\hline 17 & & & & 3.009 & 63 & & & & & & & & & \\
\hline 18 & & & & 3.041 & $64^{\mathrm{a}}$ & 46 & & & & & & & & \\
\hline & 998 & 076 & 082 & 3.085 & 65 & 2.994 & & 3.008 & & $111^{\mathrm{a}}$ & & & & \\
\hline 20 & & & & .069 & 66 & & & & & & & & & \\
\hline 21 & & & & & 67 & & & & & & & & & \\
\hline & & & & 9 & 68 & & & .104 & & & & & & \\
\hline & & & & & 69 & & & & & & & & & \\
\hline 24 & & & & 983 & 70 & & & & & 116 & & & & \\
\hline 25 & & & & & $71^{\mathrm{a}}$ & & & & & & & & & \\
\hline & & & & & 72 & & & & & & & & & \\
\hline & & & & & 13 & & & & & & & & & \\
\hline 28 & & & & & 7 & & & & & & & & & \\
\hline 2 & & & & & & & & & & & & & & \\
\hline & & & & 032 & $76^{\mathrm{a}}$ & & & & & $122^{\mathrm{a}}$ & & & & \\
\hline 31 & & & & & $77^{\mathrm{a}}$ & & & & & & & & & \\
\hline 32 & & & & & $78^{\mathrm{a}}$ & & & & & & & & & \\
\hline & & & & & 79 & & & & & 25 & & & & \\
\hline & & & & & 80 & & & & & & & & & \\
\hline & & & & 965 & 81 & 56 & 980 & 2.978 & 2.96 & $127^{\mathrm{a}}$ & 876 & & & \\
\hline & 27 & 018 & 021 & 007 & $82^{\mathrm{a}}$ & 2 & & 966 & & 128 & & & & \\
\hline $37^{\mathrm{a}}$ & & & & .047 & $83^{\mathrm{a}}$ & & & & 3.0 & 129 & & & & \\
\hline 38 & & & & & 84 & & & & & & & & & \\
\hline 3 & & & & & & & & & & & & & & \\
\hline & & & & & & & & & & & & & & \\
\hline 4 & & & & & $87^{\mathrm{a}}$ & & & & 3.2 & & 76 & & & \\
\hline & & & & & 88 & & & & & & & & & \\
\hline 43 & & & & & & & & & & & & & & \\
\hline 4 & & & & & 0 & & & & 3. & $136^{\mathrm{a}}$ & & & 10 & 3.11 \\
\hline 45 & & & & & $91^{\mathrm{a}}$ & & & & & 137 & & & & \\
\hline & & & & 93 & 92 & & & & & 138 & 3.1 & & & \\
\hline
\end{tabular}

Internal validation

\section{${ }^{a}$ Test Set}

\section{Cross-Validation}

The cross-validation statistical procedure can be used to evaluate the predictive power of QSRR models. The leave-one-out (LOO) procedure successively removes one molecule from the training set containing n molecules. A QSRR model is constructed on a " $\mathrm{n}-1$ " set of compounds and the molecule removed is predicted by the model. This procedure is repeated " $\mathrm{n}$ " times in order to predict the retention of all molecules.

The QSRR models expressed by the equations of MLR and MNLR are validated by its appreciable values of $r_{C V}{ }^{2}$ (Table 5) obtained using the LOO procedure. The value of $r_{C V}{ }^{2}$ greater than 0.5 is the basic condition for qualifying a QSRR model as valid.

Table 5. $\mathrm{r}_{\mathrm{CV}}{ }^{2}$ values obtained by the LOO

\begin{tabular}{lll}
\hline & MLR & MNLR \\
\hline $\mathrm{r}_{\mathrm{CV}}$ & 0.890 & 0.883 \\
$\mathrm{r}_{\mathrm{CV}}{ }^{2}$ & 0.792 & 0.780 \\
\hline
\end{tabular}


We use cross validation as an internal test of the quality of MLR and MNLR models. The performance of models was good and was characterized by $\mathrm{r}_{\mathrm{CV}}{ }^{2}$ values; 0.792 for the MLR and 0.780 for MNLR method (Table 5).

\section{$y$-Randomization test}

To ensure that the developed models are robust and not derive due to chance, the $y$ randomization test was performed on the training set data as recommended ${ }^{18}$. In this test, models are generated by randomly scrambling the dependent variable $(\log (\operatorname{LRI}))$ while keeping the independent variable (descriptors) unchanged. The resulting models are expected to have significantly low $\mathrm{r}^{2}$ and cross validated $\mathrm{r}_{\mathrm{CV}}{ }^{2}$ values for several trials, which confirm that the developed models are robust. We performed 100-y-randomization tests and observed that for all the models, the values of $\mathrm{r}^{2}$ and $\mathrm{r}_{\mathrm{CV}}{ }^{2}$ were $<0.5$ (Figure 5). This test confirms that the developed models are robust and not derived merely due to chance ${ }^{19}$.

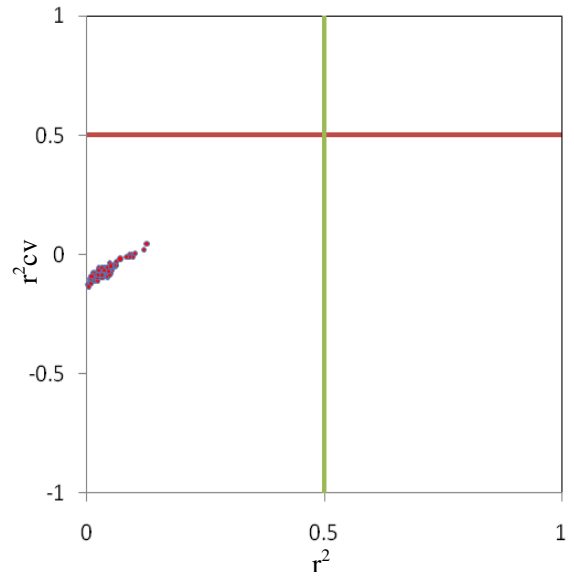

MLR model

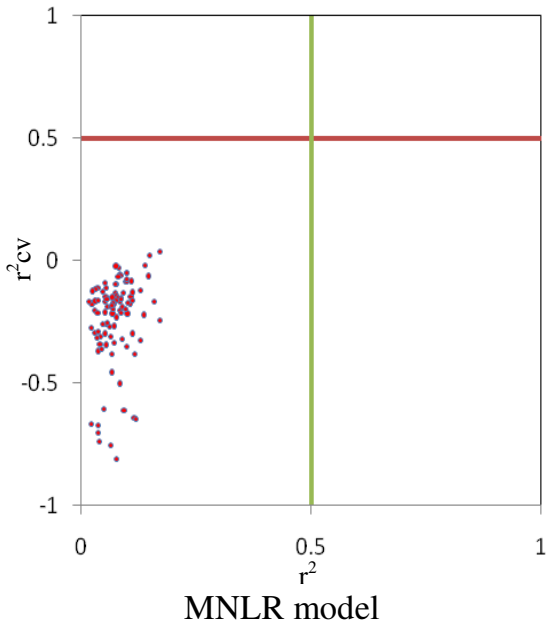

MNLR model

Figure 5. $y$-Randomization plot of MLR and MNLR models

\section{External validation}

To estimate the predictive power of the MLR, MNLR and ANN models, we have used a set of compounds that have not been used in the training set to establish the models. The equations of the models established are used to predict the linear retention indices $(\log (\mathrm{LRI}))$ of the remaining (41 molecules). The main performance parameters for the three models are shown in Table 6.

Table 6. Comparison of MLR, MNLR and ANN models

\begin{tabular}{ccccc}
\hline & & MLR & MNLR & ANN \\
\hline \multirow{4}{*}{ Training Set } & $r$ & 0.900 & 0.908 & 0.903 \\
& $r^{2}$ & 0.810 & 0.824 & 0.816 \\
& $M S E$ & 0.003 & 0.002 & 0.002 \\
Test Set & $r$ & 0.903 & 0.899 & 0.909 \\
& $r^{2}$ & 0.816 & 0.804 & 0.826 \\
& $M S E$ & 0.002 & 0.003 & 0.002 \\
\hline
\end{tabular}

The results obtained by the three models, are very sufficient to conclude the performance of models; it's confirmed by the test done with the 41 compounds. 
A comparison of the quality of MLR, MNLR and ANN models shows that the three approaches have better predictive capability gives better results. MLR, MNLR and ANN were able to establish a satisfactory relationship between the molecular descriptors and the linear retention indices $(\log (\mathrm{LRI}))$ of the studied compounds.

\section{Domain of applicability}

Evaluation of the applicability domain of the QSRR model is considered as an important step to establish that the model is reliable to make predictions within the chemical space for which it was developed ${ }^{20}$. There are several methods for defining the applicability domain of a QSRR model, but we used the most commonly used leverage approach in this study ${ }^{21}$. Leverage of a given chemical compound hi is defined as:

$$
\mathrm{h}_{\mathrm{i}}=\mathrm{x}_{\mathrm{i}}^{\mathrm{T}}\left(\mathrm{X}^{\mathrm{T}} \mathrm{X}\right)^{-1} \mathrm{x}_{\mathrm{i}}(\mathrm{i}=1 \ldots \mathrm{n})
$$

Where $\mathrm{x}_{\mathrm{i}}$ is the descriptor row of the query compound and $\mathrm{X}$ is the descriptor matrix of the training set compounds used to develop the model. As a prediction tool, the warning leverage $\mathrm{h} *$ is defined as:

$$
\mathrm{h}^{*}=3(\mathrm{p}+1) / \mathrm{n}
$$

Where $\mathrm{n}$ is the number of training compounds and $\mathrm{p}$ is the number of descriptors in the model. The test compounds with leverages $\mathrm{hi}<\mathrm{h}^{*}$ are considered to be reliably predicted by the model. The Williams plot is used to interpret the applicability domain of the model. The domain of reliable prediction for external test set compounds is defined as compounds which have leverage values within the threshold $\left(\mathrm{hi}<\mathrm{h}^{*}\right)$ and standardized residuals no greater than 3 units $( \pm \delta)$. Test set compounds where $\left(\mathrm{hi}>\mathrm{h}^{*}\right)$ are considered to be unreliably predicted by the model due to substantial extrapolation. For the training set, the Williams plot is used to identify compounds with the greatest structural influence $\left(\right.$ hi $\left.>h^{*}\right)$ in developing the model.

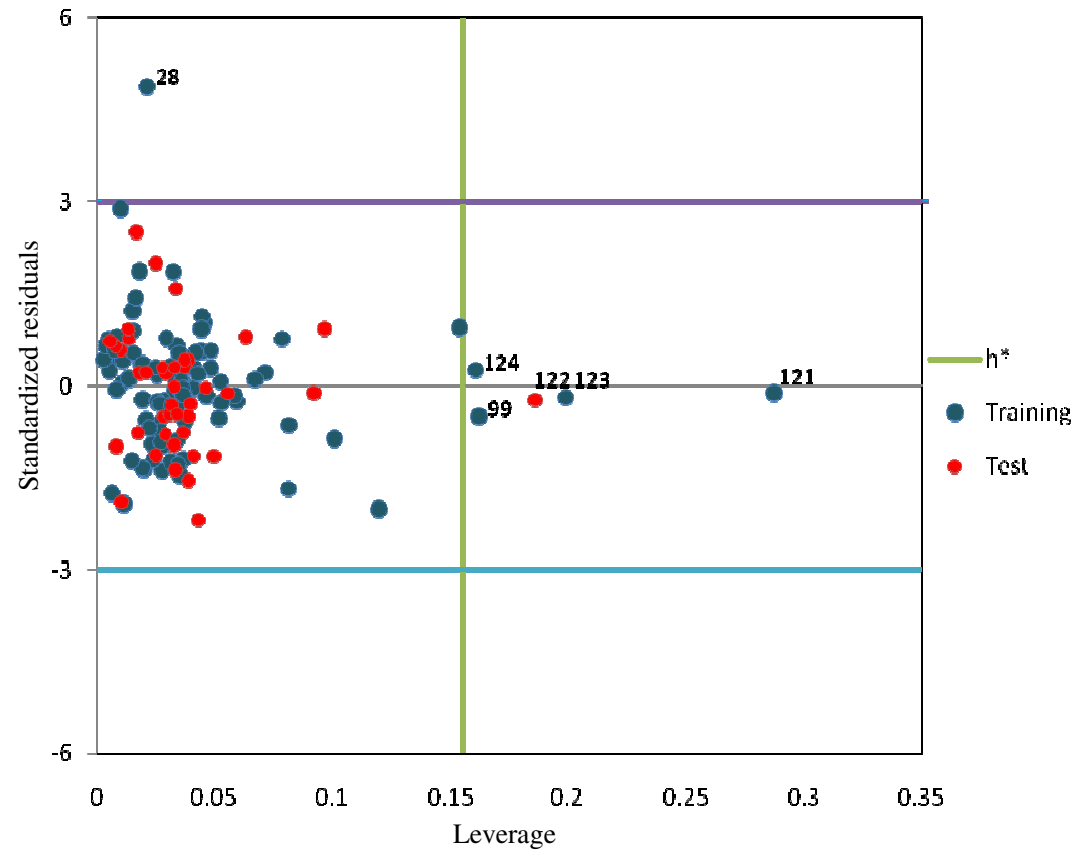

Figure 6. Williams plot to evaluate the applicability domain of MLR model 
From the Williams plot (Figure 6), it is obvious that all the compounds in the dataset are within the applicability domain of the model (the warning leverage limit is 0.155 ) except one test set compound (122) and four training compounds (99, 121, 123 and 124), these four compounds have their leverage values greater than the warning $\mathrm{h}^{*}$ value and could be high leverage compounds influencing the performance of the model. However, their standard residual values are very low and within the established limit ${ }^{17}$. As a result, these four compounds could be considered as influential in fitting the model performance but not necessarily outliers to be deleted from the training dataset and thus the model can be applied with confidence within the defined applicability domain.

For all the compounds in the training and test sets, their standardized residuals are smaller than three standard deviation units $(3 \pm \delta)$, except one in the training set (compound $\left.\mathrm{N}^{\circ} 28\right)$. Therefore, the predicted linear retention indices ( $\left.\log (\mathrm{LRI})\right)$ by the developed MLR model is reliable

\section{Proposed novel compounds}

QSRR correlates retention data with the physicochemical properties of a group of compounds. It has been frequently used to predict proprieties of new compounds and to design compounds with desired properties.

The developed Eq. 1 can be used for the designing of new volatile compounds derivatives with improved linear retention indices(Log(LRI)).

Comparing $t$-test and standardized coefficient values of descriptors (Table 7) indicates that the influences of the Critical temperature (CT), the Partition coefficient (PC) and the number of $\mathrm{H}$-Bond donors (NHD) on $\log (\mathrm{LRI})$ are stronger than this of the Percent ratios of carbon $(\mathrm{C} \%)$.

Table 7. T-test and standardized coefficient values of descriptors for equation (1)

\begin{tabular}{cccc}
\hline & $\begin{array}{c}\text { Standardized } \\
\text { coefficient }\end{array}$ & $t$-test & Sign. \\
\hline CT & 0.000 & 17.620 & $<\mathbf{0 . 0 0 0 1}$ \\
PC & 0.003 & -4.534 & $<\mathbf{0 . 0 0 0 1}$ \\
C\% & 0.044 & 2.905 & 0.005 \\
NHD & 0.012 & 7.190 & $<\mathbf{0 . 0 0 0 1}$ \\
\hline
\end{tabular}

The equation (1) of the MLR method indicated the positive correlation of the critical temperature (CT) and the number of H-Bond donors (NHD), it indicated also the negative correlation of the partition coefficient (PC).

The obtained results show that, to increase linear retention indices of volatile compounds, we will increase critical temperature (CT) and the number of $H$-Bond donors (NHD) and we will decrease the partition coefficient (PC). Moreover, to decrease linear retention indices, we will decrease critical temperature (CT) and the number of $H$-Bond donors (NHD) and we will increase the partition coefficient (PC) of this molecules, by adding suitable substituent's and calculated their linear retention indices $(\log (\mathrm{LRI}))$ using the Eq. 1.

The structures of the designed compounds and their parameter values calculated by the same methods as well as $\log (\mathrm{LRI})$ values theoretically predicted by the MLR model (Eq. 1) are listed in Table 8 . 
Table 8. Values of descriptors, linear retention indices (Log(LRI)) and leverages (h) for the new designed compounds

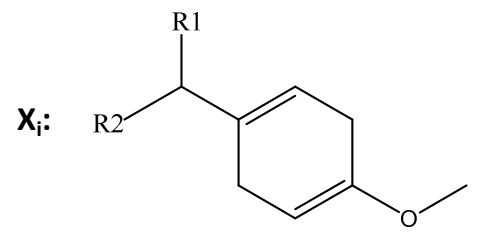

\begin{tabular}{|c|c|c|c|c|c|c|c|}
\hline & Designed compounds & $\mathrm{CT}$ & $\mathrm{PC}$ & $\mathrm{C} \%$ & NHD & $\log (\mathrm{LRI})$ & Leverage, $\mathrm{h}$ \\
\hline $\mathrm{X}_{1}$ & $\mathrm{R} 1=\mathrm{OH} ; \mathrm{R} 2=\mathrm{CH}_{3}$ & 692.56 & 0.676 & 0.701 & 1 & 3.310 & 0.050 \\
\hline $\mathrm{X}_{2}$ & $\mathrm{R} 1=\mathrm{OH} ; \mathrm{R} 2=\mathrm{OH}$ & 719.10 & -0.462 & 0.615 & 2 & 3.445 & 0.163 \\
\hline$X_{3}$ & $\mathrm{R} 1=\mathrm{OH} ; \mathrm{R} 2=\mathrm{C}_{2} \mathrm{H}_{5}$ & 706.75 & 1.205 & 0.714 & 1 & 3.328 & 0.054 \\
\hline $\mathrm{X}_{4}$ & $\mathrm{R} 1=\mathrm{OH} ; \mathrm{R} 2=\mathrm{CH}\left(\mathrm{CH}_{3}\right)_{2}$ & 710.14 & 1.604 & 0.725 & 1 & 3.330 & 0.055 \\
\hline $\mathrm{X}_{5}$ & $\mathrm{R} 1=\mathrm{OH} ; \mathrm{R} 2=\mathrm{C}_{3} \mathrm{H}_{7}$ & 719.95 & 1.734 & 0.725 & 1 & 3.344 & 0.060 \\
\hline $\mathrm{X}_{6}$ & $\mathrm{R} 1=\mathrm{CH}_{2} \mathrm{OH} ; \mathrm{R} 2=\mathrm{CH}_{3}$ & 714.14 & 1.095 & 0.714 & 1 & 3.341 & .059 \\
\hline $\mathrm{X}_{7}$ & $\mathrm{R} 1=\mathrm{CH}_{2} \mathrm{OH} ; \mathrm{R}_{2}=\mathrm{CH}_{2} \mathrm{OH}$ & 754.84 & -0.236 & 0.562 & 2 & 3.493 & 183 \\
\hline $\mathrm{X}_{8}$ & $\mathrm{R} 1=\mathrm{CH}_{2} \mathrm{OH} ; \mathrm{R} 2=\mathrm{C}_{2} \mathrm{H}_{5}$ & 726.86 & 1.624 & 0.725 & 1 & 3.356 & 0.064 \\
\hline $\mathrm{X}_{9}$ & $\mathrm{R} 1=\mathrm{CH}_{2} \mathrm{OH} ; \mathrm{R} 2=\mathrm{CH}\left(\mathrm{CH}_{3}\right)_{2}$ & 729.84 & 2.023 & 0.734 & 1 & 3.357 & 0.066 \\
\hline $\mathrm{X}_{10}$ & $\mathrm{R} 1=\mathrm{CH}_{2} \mathrm{OH} ; \mathrm{R} 2=\mathrm{CH}\left(\mathrm{CH}_{3} \mathrm{OH}\right)$ & 752.14 & 0.073 & 0.666 & 2 & 3.498 & 0.181 \\
\hline $\mathrm{X}_{11}$ & $\mathrm{R} 1=\mathrm{CH}_{2} \mathrm{OH} ; \mathrm{R} 2=\mathrm{C}_{3} \mathrm{H}_{7}$ & 738.77 & 2.153 & 0.734 & 1 & 3.370 & 0.071 \\
\hline $\mathrm{X}_{12}$ & $\mathrm{R} 1=\mathrm{CH}_{2} \mathrm{OH} ; \mathrm{R} 2=\mathrm{Cl}$ & 740.90 & 0.759 & 0.573 & 1 & 3.371 & 0.081 \\
\hline $\mathrm{X}_{13}$ & $\mathrm{R} 1=\mathrm{CH}_{2} \mathrm{OH} ; \mathrm{R} 2=\mathrm{F}$ & 694.69 & 0.419 & 0.628 & 1 & 3.307 & 0.051 \\
\hline $\mathrm{X}_{14}$ & $\mathrm{R} 1=\mathrm{CH}_{2} \mathrm{OH} ; \mathrm{R} 2=\mathrm{Br}$ & 762.31 & 0.899 & 0.464 & 1 & 3.389 & 0.116 \\
\hline $\mathrm{X}_{15}$ & $\mathrm{R} 1=\mathrm{OH} ; \mathrm{R} 2=\mathrm{Br}$ & 745.15 & 1.120 & 0.439 & 1 & 3.356 & 0.111 \\
\hline $\mathrm{X}_{16}$ & $\mathrm{R} 1=\mathrm{OH} ; \mathrm{R} 2=\mathrm{C}_{6} \mathrm{H}_{5}$ & 799.07 & 2.015 & 0.778 & 1 & 3.475 & 0.121 \\
\hline$X_{17}$ & $\mathrm{R} 1=\mathrm{CH}_{2} \mathrm{OH} ; \mathrm{R} 2=\mathrm{C}_{6} \mathrm{H}_{5}$ & 810.61 & 2.134 & 0.782 & 1 & 3.492 & 0.131 \\
\hline
\end{tabular}

From the predicted properties, it has been observed that the designed compounds $\mathrm{X}_{1}$, $\mathrm{X}_{2}, \mathrm{X}_{3}, \mathrm{X}_{4}, \mathrm{X}_{5}, \mathrm{X}_{6}, \mathrm{X}_{7}, \mathrm{X}_{8}, \mathrm{X}_{9}, \mathrm{X}_{10}, \mathrm{X}_{11}, \mathrm{X}_{12}, \mathrm{X}_{13}, \mathrm{X}_{14}, \mathrm{X}_{15}, \mathrm{X}_{16}$ and $\mathrm{X}_{17}$ have higher Log(LRI) values than the existing compounds in the case of the 138 studied compounds (Table 1).

The leverage values (h) calculated by Eq. 1 of the MLR for the new designed compounds are displayed in Table 8. Only three compounds $\mathrm{X}_{2}, \mathrm{X}_{7}$ and $\mathrm{X}_{10}$ are defined as outliers and consequently they are not be considered, because they have higher leverage which is greater than $\mathrm{h}^{*}\left(\mathrm{~h}^{*}=0.155\right)^{11}$.

\section{Conclusion}

In this study, multiple linear and non-linear regression and artificial neural networks were used to construct quantitative structure retention relation models of volatile compounds for their linear retention indices (LRI). MLR analysis produced more predictive, informative and significantly improved QSRR model. All QSRR models provide a reasonably good correlation coefficient $(r \geq 0.900)$. The validation and predictive ability of the models were examined by the leave one-out cross-validation, $Y$-randomization and external validation. The three methods indicated that the resulting multiparametric QSRR models possess high prediction ability and low over fitting. The applicability domain of the MLR model was defined.

We conclude that the most important finding about this research is that we have been able to design and proposed some new compounds with high linear retention indices (LRI) values than the existing ones by adding suitable substituent's and calculated their linear retention indices using regression equation. 


\section{Acknowledgment}

We are grateful to the "Association Marocaine des ChimistesThéoriciens" (AMCT) for its pertinent help concerning the programs.

\section{References}

1. Ghavami R and Faham S, Chromatographia, 2010, 72(9-10), 893-903; DOI:10.1365/s10337-010-1741-4

2. Dai J, Jin L, Yao S H and Wang L, Chemosphere, 2001, 42(8), 899-907. DOI:10.1016/S0045-6535(00)00181-8

3. Qin L, Liu Sh Liu H and Tong C, J Chromatogr A, 2009, 1216(27), 5302-5312. DOI:10.1016/j.chroma.2009.05.016

4. Kaliszan R, Recent advances in quantitative structure retention relationships; separation methods in drug synthesis and purification. In: Valko K, (Ed), Elsevier, Amsterdam, 2000.

5. Hu R J, Liu H X, Zhang R S, Xue C X, Yao X J, Liu M C, Hu Z D and Fan B T, Talanta, 2005, 68(1), 31-39; DOI:10.1016/j.talanta.2005.04.034

6. López-Pérez O, Picon A and Nuñez M, Food Res Int., in press; 2016, 99(3), 10021010; DOI:10.1016/j.foodres.2016.12.013

7. SPSS 19.0, http://www.ibm.com/analytics/fr/fr/technology/spss/.

8. ACDLABS 10, "Advanced Chemistry Development, Inc., Toronto, ON, Canada," 2015, http://www.acdlabs.com

9. Marvin Sketch 5.11.4. "Chem Axon.” 2012, http://www.chemaxon.com

10. ChemBioOffice, PerkinElmer Informatics, 2010, http://www.cambridgesoft.com

11. Chtita S, Ghamali M, Hmamouchi R, Elidrissi B, Bourass M, Larif M, Bouachrine M and Lakhlifi T, Adv Phys Chem., 2016, 1-16. DOI:10.1155/2016/5137289

12. Gramatica P, Comb Sci., 2007, 26(5), 694-701; DOI:10.1002/qsar.200610151

13. Belhassan A, Chtita S, Lakhlifi T and Bouachrine M, Electro J Chem., 2017, 9(4), 234-237; DOI:10.17807/orbital.v9i4.978

14. Chtita S, Hmamouchi R, Larif M, Ghamali M, Bouachrine M and Lakhlifi T, $J$ Taibah Univ Sci., 2016, 10(6), 868-876; DOI:10.1016/j.jtusci.2015.04.007.

15. Belhassan A, Chtita S, Lakhlifi T and Bouachrine M, J Taibah Univ Sci., 2017, 11(6), 1030-1046; DOI:10.1016/j.jtusci.2017.05.004.

16. Chtita S, Larif M, Ghamali M, Bouachrine M and Lakhlifi T, J Taibah Univ Sci., 2015, 9(2), 143-154; DOI:10.1016/j.jtusci.2014.10.006.

17. Bastani D, Hamzehie M E, Davardoost F, Mazinani S and Poorbashiri A, Fluid Phase Equilibria, 2013, 354, 6-11; DOI:10.1016/j.fluid.2013.05.017

18. Roy K and Mitra I, Comb Chem High Throughput Screen, 2011, 14(6), 450-474; DOI:10.2174/138620711795767893

19. Garg R and Smith C J, Food Chem Toxicol., 2014, 69, 252-259; DOI:10.1016/j.fet.2014.03.035

20. Eriksson L, Jaworska J, Worth A P, Cronin M T D, McDowell R M and Gramatica P, Environ Health Perspect, 2003, 111(10), 1361-1375; http://refhub.elsevier.com/S0278-6915(14)00164-1/h0065

21. Gramatica P, Comb Sci., 2007, 26, 694-701; http://refhub.elsevier.com/S02786915(14)00164-1/h0085 